\title{
Retinoic acid signaling and neurogenic niche regulation in the developing peripheral nervous system of the cephalochordate amphioxus
}

Elisabeth Zieger ${ }^{1}$, Greta Garbarino ${ }^{2}$, Nicolas S. M. Robert ${ }^{1}$, Jr-Kai Yu³ ${ }^{3}$, Jenifer C. Croce $^{1}$, Simona Candiani ${ }^{2}$, Michael Schubert ${ }^{1 *}$

\begin{abstract}
${ }^{1}$ Sorbonne Universités, UPMC Université Paris 06, CNRS, Laboratoire de Biologie du Développement de Villefranche-sur-Mer, Observatoire Océanologique de Villefranche-surMer, 181 Chemin du Lazaret, 06230 Villefranche-sur-Mer, France.

${ }^{2}$ Department of Earth, Environment and Life Sciences (Dipartimento di Scienze della Terra dell'Ambiente e della Vita, DISTAV), University of Genoa, Viale Benedetto XV 5, 16132 Genoa, Italy.
\end{abstract}

${ }^{3}$ Institute of Cellular and Organismic Biology, Academia Sinica, Taipei 11529, Taiwan.

${ }^{*}$ Corresponding author:

ORCID:

0000-0002-2341-712X

Email:

michael.schubert@obs-vlfr.fr

Tel:

+ 33 (0) 493763791

Fax:

+ 33 (0) 493763792

Web:

http://lbdv.obsvlfr.fr/en/research/research_groups/evolution_of_intercellular_signaling_in_dev elopment_evoinside.html 


\begin{abstract}
The retinoic acid (RA) signaling pathway regulates axial patterning and neurogenesis in the developing central nervous system (CNS) of chordates, but little is known about its roles during peripheral nervous system (PNS) formation and about how these roles might have evolved. This study assesses the requirement of RA signaling for establishing a functional PNS in the cephalochordate amphioxus, the best available stand-in for the ancestral chordate condition. Pharmacological manipulation of RA signaling levels during embryogenesis reduces the ability of amphioxus larvae to respond to sensory stimulation and alters the number and distribution of ectodermal sensory neurons (ESNs) in a stage- and contextdependent manner. Using gene expression assays combined with immunohistochemistry, we show that this is because RA signaling specifically acts on a small population of Soxb1c-expressing ESN progenitors, which form a neurogenic niche in the trunk ectoderm, to modulate ESN production during elongation of the larval body. Our findings reveal an important role for RA signaling in regulating neurogenic niche activity in the larval amphioxus PNS. Although only few studies have addressed this issue so far, comparable RA signaling functions have been reported for neurogenic niches in the CNS and in certain neurogenic placode derivatives of vertebrates. Accordingly, the here-described mechanism is likely a conserved feature of chordate embryonic and adult neural development.
\end{abstract}

Keywords: evolution of development, lancelet, neural stem cells, retinoid pathway, sensory functions 


\section{Introduction}

In order to successfully interact with their environment, most multicellular animals require a peripheral nervous system (PNS) that receives and transmits sensory information as well as instructive signals from the central nervous system (CNS). In vertebrates, miss-regulation of physiological retinoic acid (RA) levels during development is associated with various behavioral defects, such as hyperactivity [1], decreased social affiliation [2], impaired vestibular and motor functions [3, 4], and intellectual disability [5]. Consistently, RA signaling has been shown to contribute to the formation of various PNS structures and sensory organs, including the eyes [69], the olfactory epithelium [10,11], the otocyst that gives rise to components of the inner ear [12-14], and the enteric nervous system [15]. These data strongly suggest that the RA signaling pathway is crucially involved in overall PNS development, but information on its precise roles or on how they might have emerged in the course of animal evolution remains fragmentary $[16,17]$.

RA signals are chiefly transduced by heterodimers of two nuclear hormone receptors, the $R A$ receptor (RAR) and the retinoid $X$ receptor (RXR). These RAR/RXR heterodimers bind directly to specific DNA motifs, called retinoic acid response elements (RAREs) [18, 19]. In the absence of a ligand, the association of RAR/RXR heterodimers with a RARE generally leads to the recruitment of corepressor complexes that mediate chromatin compaction and target gene repression [20]. Fixation of RA activates the ligand-dependent transcription factor function of the RAR/RXR heterodimer, which causes dissociation of the co-repressor complex and recruitment of a co-activator complex, thus ultimately inducing target gene transcription [21, 22].

Initially believed to be vertebrate-specific, RA has now been implicated in the neural development of various other taxa, including cnidarians [23], gastropod mollusks [24-26], tunicates [27, 28], and cephalochordates [29-32]. The latter are a slow evolving taxon that forms the sister group of all other chordates, which further include tunicates and vertebrates [33-37]. Since cephalochordates, commonly called amphioxus, have not undergone the two rounds of whole genome duplications that are typical of vertebrates [38-40], they possesses only a single rar and a single rxr 
gene, while vertebrate genomes generally encode three $\operatorname{rar}(\operatorname{rar} \alpha, \operatorname{rar} \beta$, and $\operatorname{rar} \gamma)$ and three $r \times r(r \times r \alpha, r \times r \beta$, and $r \times r \gamma)$ genes [16]. Even so, it has been demonstrated that, as in vertebrates, the amphioxus RAR/RXR heterodimer can be activated by binding RA, which is necessary for establishing collinear hox gene expression patterns and for axial patterning of the nervous system [29, 31, 32, 41-43].

This is in agreement with studies showing that the CNS of amphioxus is subdivided into several territories that display homology with structures of the vertebrate CNS, including a combined dien-mesencephalon, a hindbrain, and a spinal cord [39,44-46]. Despite these similarities, the adult amphioxus CNS is much smaller and less complex than that of vertebrates. It comprises only about 20.000 neural cells and exhibits no external structuring, apart from a caudal ampulla and serially repeated dorsal nerves that lack ganglia [45]. Taken together, these findings show that both the RA signaling cascade and the nervous system of amphioxus are simpler than those of vertebrates, but still highly comparable. Accordingly, cephalochordates are attractive models for studying the evolution of RA signaling functions and chordate neural development [16, 32, 40, 47, 48].

Apart from a dorsal neuroectoderm that undergoes neurulation to form the CNS, amphioxus embryos possess a second neurogenic domain in the ventral ectoderm, which gives rise to the PNS [49, 50]. This ventral neurogenic domain, chiefly referred to as the ectodermal sensory neuron (ESN) precursor field, is thought to be related to vertebrate cranial placodes and becomes specified at the late gastrula stage, when the homeobox transcription factor $t / x$ (T-cell leukemia homeobox) starts to be expressed along the midline of the ventral ectoderm [39, 50, 51]. Initially, $t / x$ is widely expressed, but gradually becomes restricted to individual ESN progenitors (ESNPs) that delaminate into the sub-ectodermal space to migrate dorsally [49]. At the flanks of the embryo, the ESNPs downregulate t/x expression, reinsert their soma into the ectoderm and differentiate [49]. Notably, the number of ESNs has been reported to increase during subsequent larval development [45], but it is currently unclear whether the migrating ESNPs are able to divide or if another neurogenic niche for the generation of peripheral neurons exists in the amphioxus larva. 
Different types of the ESNPs can be distinguished according to their expression of distinct sets of neural marker genes, which become induced during their migration. Both $t / x$ and hu/elav are probably expressed in all ESNPs, but $t / x$ expression is eliminated from most cells as they differentiate $[49,52]$. Other genes, such as eya, six1/2, soxb1c, err, islet, coe, and different hox genes, are only expressed in specific subsets of ESNPs, with eya, six1/2, and soxb1c being confined to late-developing ESNPs in the mid-trunk ectoderm [29, 51, 53, 54]. On a morphological level, two main types of ESNs are discernable. Primary type I ESNs develop early in the rostrum and tail, from where their axons enter the neural tube and travel long distances within it, to make repeated synaptic contacts with ventral interneurons involved in locomotory control [45, 55]. More specialized type I ESNs may exhibit a modified, spine-like cilium, whereas type II ESNs possess a collar of branched microvilli and probably function as chemoreceptors [45]. In addition, amphioxus ESNs may exhibit different neurotransmitter phenotypes, including mostly glutamatergic, but also GABAergic cells [56]. It is currently unclear how these different types of ESNs become specified, but the RA signaling pathway is likely to be involved, since it regulates amphioxus hox gene expression in the ectoderm and was shown to influence the anterior-posterior positioning of ESNPs expressing the neural marker genes err, islet, and coe [29].

Given the general importance of RA signaling for neurobehavioral and PNS development in vertebrates, we decided to investigate how the RA pathway contributes to these processes in amphioxus (Branchiostoma lanceolatum). We thus first altered RA signaling levels at selected embryonic stages using pharmacological treatments with the RAR antagonist BMS493 and with exogenous all-trans RA. Next, we examined proper functioning of the amphioxus PNS through behavioral tests for mechano- and chemoreception. The obtained data show that disruption of endogenous RA signaling levels reduces the ability of amphioxus larvae to respond to sensory stimulation in a stage-dependent manner. In order to determine, whether this is due to defective PNS formation, we documented neurogenesis of ESNs through immunohistochemistry against the neuronal marker acetylated tubulin (AT) and the very abundant neurotransmitter glutamate (GLU). We found that RA signaling levels act in a strictly stage-dependent manner to regulate the number and distribution of ESNs. In an effort to elucidate, how this was achieved, we used 
proliferation and apoptosis assays as well as in situ hybridization approaches targeting the neural marker genes hu/elav, $t / x$, and soxb1c. Importantly, our results reveal that RA signaling mainly acts on a small late-developing population of soxb1c expressing ESNPs in the trunk ectoderm to modulate ESN production. This neurogenic niche is most likely responsible for supplying new ESNs as the amphioxus larva elongates. Comparable RA signaling functions have previously been reported in neurogenic niches of both the vertebrate CNS and PNS, suggesting that this mechanism is evolutionary conserved among chordates.

\section{Methods and materials}

\section{Animals}

European amphioxus (B. lanceolatum) adults were collected from sand samples retrieved in Argelès-sur-Mer, France. About 10 animals were kept in 5 I aquaria with a water temperature of 16 to $17^{\circ} \mathrm{C}$ and a periodical light cycle of $14 \mathrm{hrs}$ light and 10 hrs darkness. To induce spawning, selected males and females were exposed to a $36-\mathrm{hr}$ thermal shock at $23^{\circ} \mathrm{C}$, as previously described [57-59]. From each female, several hundred eggs were collected in a Petri dish. After successful in vitro fertilization, the developing zygotes were incubated in artificial seawater (Red Sea Salt, Verneuil-sur-Avre, France) at $19^{\circ} \mathrm{C}$ in the dark [60].

\section{Pharmacology}

Embryos of $B$. lanceolatum were treated with the RAR antagonist BMS493 at a concentration of $1 \mu \mathrm{M}$ or with all-trans $\mathrm{RA}$ at a concentration of $0.1 \mu \mathrm{M}$. Both compounds were purchased from Sigma-Aldrich (Saint-Quentin Fallavier, France) and were dissolved in dimethyl sulfoxide (DMSO) (Sigma-Aldrich, Saint-Quentin Fallavier, France) to create $1000 \mathrm{X}$ stock solutions, which were added to $10 \mathrm{ml} \mathrm{B}$. lanceolatum embryo cultures, at the respective final concentrations. In addition, control cultures were treated with DMSO at a final concentration of 1:1000 [41]. 
Pharmacological treatments began either at the late blastula (6 hpf), mid-gastrula (12 hpf) or mid-neurula (24 hpf) stage.

\section{Behavioral analyses}

For each behavioral experiment, amphioxus larvae at $48 \mathrm{hpf}$ were transferred into a Petri dish containing $10 \mathrm{ml}$ (mechanoreception) or $2.5 \mathrm{ml}$ (chemoreception) of artificial seawater. Next, the animals were left under a stereomicroscope to acclimatize for $15 \mathrm{~min}$ and their behavior was filmed using a Hamamatsu Flash4 camera. To test for mechanoreception, larvae were gently touched with the tip of a plastic Pasteur pipette (Supplementary file 1). To create a small flexible tip that could be used to touch the larvae without damaging them, the thin end of a plastic Pasteur pipette was heated over a low flame and slowly elongated until its diameter was about $0.15 \mathrm{~mm}$. Before use, the pipette was washed several times with artificial seawater. For each treatment condition, $n=50$ larvae were filmed and their responses were classified and counted (Fig. 1). To test for chemoreception, a small block of low-melting agarose (5 x 5 × $5 \mathrm{~mm}$ ) (Sigma-Aldrich, Saint-Quentin Fallavier, France), dissolved in either artificial seawater (negative control) or in artificial seawater containing $0.1 \mathrm{M}$ L-glutamate, was placed into a Petri dish containing $2.5 \mathrm{ml}$ of artificial seawater and 50 amphioxus larvae (Fig. 2a). Subsequently, the larvae were filmed for $10 \mathrm{~min}$ and their movements were tracked using the MTrackJ plugin of the ImageJ software (Fig. 2a) [61]. For each treatment condition, the experiment was repeated 5 times and the behavior of $n=50$ larvae, which passed the agarose block within a $1 \mathrm{~cm}$ radius and within 1 to $10 \mathrm{~min}$ after introduction of the agarose block, were classified and counted (Fig. 2b,c; Supplementary file 2). Furthermore, the number of circles a larva would swim without interruption was counted for as many animals as possible ( $n=$ indicated in Additional file 3: Figure S1).

\section{Sequence identification, phylogenetic analyses, and gene cloning}

The sequences for hu/elav, $t / x$, and soxb1c were identified in the available $B$. lanceolatum sequence data [62], through tBLASTn searches using as queries the 
Homo sapiens protein sequences of ELAV1, ELAV2, ELAV3, ELAV4 (respectively, NP_001410.2, NP_004423.2, NP_001411.2, NP_068771.2), TLX1, TLX2, TLX3 (respectively, NP_005512.1, NP_057254.1, NP_066305.2), and SOX1, SOX2, SOX3 (respectively, NP_005977.2, NP_003097.1, NP_005625.2). Subsequently, the obtained candidate B. lanceolatum hu/elav, $t / x$, and soxb1c sequences were translated into proteins and the sequences of their potential orthologs were recovered from Homo sapiens, Gallus gallus, Strongylocentrotus purpuratus, Drosophila melanogaster, Tribolium castaneum, and B. floridae (for accession numbers, see Additional file 9: Table S1). All sequences were aligned using the MUSCLE program with default parameters [63]. Positions in the alignment with more than $75 \%$ gaps were removed using the $-g t=0.25$ parameter from TrimAl v1.2 [64]. The final alignment of the $15 \mathrm{HU} / E L A V$ sequences comprised 377 residues, the final alignment of the 11 TLX sequences comprised 330 residues, and the final alignment of the 13 SOX sequences comprised 384 residues. Maximum-likelihood phylogenies were inferred using RAxML with 1000 rapid bootstraps [65]. The best-suited model to each alignment was determined using Protest v3.4 [66], which yielded: $\mathrm{JTT}+\Gamma+\mathrm{I}$ for $\mathrm{HU} / \mathrm{ELAV}, \mathrm{DCmut}+\Gamma+\mid$ for $T L X$, and $D C m u t+\Gamma+I$ for SOX. The resulting trees (Additional file 8: Figure $S 6 a, b, c$ ) validated the B. lanceolatum hu/elav, $t / x$, and soxb1c sequences as true homologs of their $B$. floridae counterparts.

RNA was extracted from $B$. lanceolatum embryos and larvae at different developmental stages $(18,24,36$, and $48 \mathrm{hpf})$, according to established protocols [67] and cDNA was synthesized using the SuperScriptlll reverse transcription kit from Invitrogen (Cergy Pontoise, France). Next, open reading frames of the $B$. lanceolatum hu/elav (KY569299), t/x (KY569298), and soxb1c (KY569300) genes were amplified by polymerase chain reaction (PCR), using the obtained embryonic cDNA and gene-specific primers: 5'-GTTAGCGGCACAGACTCCGAAGAGCG-3' and 5'-CTGCCGTGTTTGCCCGCGTACAATTGG-3' for hu/elav, ATGGTTGGCATCATGGAATCGGAG-3' and 5 TAGCAGATCGTTGGCGTGGTGAG-3' for $t / x$,

and 5 GGTGTGGTCACGCGGACAGCGCCG-3' and 5'-CGCGCGGGAAACTTGTATCCG3 ' for soxb1c. The PCR products were cloned into the pGEM ${ }^{\circledR}-T$ Easy Vector System from Promega (Charbonnières-les-Bains, France) and the open reading frames subsequently validated by sequencing on both DNA strands. 


\section{In situ hybridization and immunohistochemistry}

For whole mount in situ hybridization and immunohistochemistry, $B$. lanceolatum embryos were fixed at different developmental stages in freshly prepared ice-cold 4\% paraformaldehyde diluted in MOPS buffer [68]. Antisense riboprobes were synthesized and in situ hybridization experiments were carried out as previously described [68]. For immunohistochemistry, fixed embryos were transferred into the wells of a 96-well plate and washed in several changes of PBS (phosphate buffered saline, $0.1 \mathrm{M}, \mathrm{pH} 7$ ) for $1 \mathrm{hr}$, followed by a pre-incubation in PBSTX (PBS plus $0.3 \%$ Triton X-100) for $1 \mathrm{hr}$. Next, the embryos were incubated in primary antibodies diluted in PBS-TX. All embryos were stained for acetylated tubulin (Sigma-Aldrich, Saint-Quentin Fallavier, France, T6793, concentration 2:1000) and glutamate (Sigma-Aldrich, Saint-Quentin Fallavier, France, G6642, concentration $3: 1000)$ for $72 \mathrm{hrs}$ at $4^{\circ} \mathrm{C}$. The primary antisera were washed off with several changes of PBS for 2 hrs and the embryos were then incubated in the secondary antibodies anti-rabbit IgG Alexa Fluor ${ }^{\circledR} 488$ (Fisher Scientific, Illkirch-Graffenstaden, France, A-11008, concentration 3:1000) and anti-mouse Cy3 ${ }^{\mathrm{TM}}$ (Bethyl Laboratories Inc., Montgomery, USA, A90-516C3, concentration 3:1000) as well as in the nucleic acid dye Hoechst (bisBenzimide, Invitrogen, Cergy Pontoise, France, concentration 1:5000), diluted in PBS, for $12 \mathrm{hrs}$ at $4^{\circ} \mathrm{C}$. Subsequently, the embryos were washed once more for $2 \mathrm{hrs}$ in several changes of PBS and mounted in Mowiol (SigmaAldrich, Saint-Quentin Fallavier, France).

\section{Proliferation apoptosis assays}

For the EdU cell proliferation assay, the Click-iT ${ }^{\circledR}$ Plus EdU Alexa Fluor ${ }^{\circledR} 647$ Imaging Kit from Fisher Scientific (Illkirch-Graffenstaden, France) was used. For EdU incorporation, B. lanceolatum neurula stage embryos (at $24 \mathrm{hpf}$ ) were exposed to $50 \mu \mathrm{M}$ EdU in artificial seawater for $4 \mathrm{hrs}$. Next, the embryos were washed with five changes of artificial seawater and left to develop until the feeding larva stage (36 hpf), when they were fixed with freshly prepared ice-cold $4 \%$ paraformaldehyde 
diluted in MOPS buffer [68]. The EdU labeling reaction was performed according to the manufacturer's description. For subsequent immunohistochemistry, EdU-labeled embryos were washed in several changes of PBS for $1 \mathrm{hr}$ and then processed as described above.

For the TUNEL apoptosis assay, the ApopTag ${ }^{\circledR}$ Peroxidase In Situ Apoptosis Detection Kit from Merck Millipore (Molsheim, France) was used. Since a strong background staining occurred in early $B$. lanceolatum embryos, larval stages (at 60 hpf) were used to detect apoptotic cells by TUNEL labeling. The whole mount detection of apoptosis in amphioxus was performed as previously described [69]. Digoxigenin-conjugated nucleotides incorporated into the free $3{ }^{\prime} \mathrm{OH}$ DNA termini of apoptotic cells were detected using the anti-digoxigenin antibody conjugated with alkaline phosphatase from Roche (Meylan, France, 11093274910). For the chromogenic reaction, Fast Red RC Tablets from Sigma-Aldrich (Saint-Quentin Fallavier, France) were used, according to manufacture's instructions. The chromogenic reaction was stopped after $10 \mathrm{~min}$ by washing the embryos in five changes of PBS and mounting them in Mowiol (Sigma-Aldrich, Saint-Quentin Fallavier, France).

\section{Imaging and image analyses}

Images of $B$. lanceolatum embryos after single in situ hybridization were taken as whole mounts using Zeiss differential interference contrast optics (Carl Zeiss SAS, Marly-le-Roi, France). All other imaging work was carried out on a Leica TCS SP5 confocal microscope (Leica Microsystems SAS, Nanterre, France). After TUNEL labeling, the Fast Red signal was excited at 514nm (Argon laser) and detected at 560 to $600 \mathrm{~nm}$. For immunostained embryos, the Су3 ${ }^{\mathrm{TM}}$ signal was excited at $543 \mathrm{~nm}$ (HeNe laser) and detected at 560 to $580 \mathrm{~nm}$, the Alexa Fluor ${ }^{\circledR} 488$ signal was excited at $488 \mathrm{~nm}$ (Argon laser) and detected at 515 to $540 \mathrm{~nm}$ and the Hoechst signal was excited at $405 \mathrm{~nm}$ (Diode laser UV) and detected at 430 to $460 \mathrm{~nm}$. For in situ hybridization/immunohistochemistry co-localization, the NBT/BCIP signal was excited at $633 \mathrm{~nm}$ (HeNe laser) and detected at 660 to $680 \mathrm{~nm}$. Series of optical sections were taken at a z-step interval of $0.8 \mu \mathrm{m}$. The ImageJ software was subsequently 
used to generate maximum projections and for further image processing [61]. Cell numbers were counted manually and statistical significance was assessed using twotailed student's t-tests assuming unequal variances. 


\section{Results}

\section{Manipulation of retinoic acid signaling levels during development negatively impacts motor- and mechanosensory functions in amphioxus larvae}

Following pharmacological treatment at either 6 or 24 hours post fertilization (hpf) with either the RAR antagonist BMS493 or exogenous all-trans RA, several aspects of larval behavior were examined. All behavioral tests were carried out at the $48 \mathrm{hpf}$ stage, when the larvae display a rich repertoire of sensory and motor functions as well as a high level of activity [70].

To test their responsiveness to mechanical stimulation, amphioxus larvae were touched laterally with the small flexible tip of a plastic pipette $(\mathrm{d}=0.3 \mathrm{~mm})$ and their reactions were filmed (Supplementary file 1). All control larvae reacted by quickly swimming away from the mechanical stimulus, using fast muscular swimming rather than slow ciliary swimming [70]. In contrast, larvae that have been subjected to alterations of endogenous RA signaling levels during their development showed a variety of different responses (Fig. 1; Supplementary file 1), which were scored as follows: (A) muscular swimming away from the stimulus, (B) intense wiggling and bending motions that resemble muscular swimming but without clear directionality, (C) short wiggling motions, (D) disconnected twitches/bends, and (E) no muscular response/ciliary swimming. For larvae that have been treated with the RAR antagonist BMS493 starting from $6 \mathrm{hpf}$, the full range of possible responses could be observed, with most animals responding in a slightly reduced fashion (reaction B, Fig. 1). Interestingly, later BMS493 treatments, at $24 \mathrm{hpf}$, led to a more severe reduction of larval responsiveness. None of the larvae swam away (response A) and half did not react at all (response E), even though they were generally capable of muscular swimming, which could be induced through more severe stimulation, e.g. by sucking animals up and down a plastic pipette several times.

\section{Fig.1}

Effects of retinoic acid (RA) signaling on mechanoreception in amphioxus larvae. Amphioxus embryos were exposed to DMSO (Control), the RA receptor (RAR) 
antagonist BMS493 or all-trans RA, starting from the treatment time points $(\mathrm{t}): 6$ or 24 hpf. For each treatment condition, the responses of $n=50$ larvae at the 48 hpf (hours post fertilization) stage to a mechanical stimulus were assessed and counted. The response A (dark green) corresponds to a quick muscular swimming movement away from the stimulus and was shown by all control animals, the response B (light green) to an intense wiggling and bending movement without clear directionality, the response $C$ (yellow) to a short wiggling motion on the spot, the response $D$ (orange) to short disconnected twitches or bends on the spot, and the response $E$ (red) to no visible movement or reaction.

When treated with RA starting from $6 \mathrm{hpf}$, the larvae were not capable of muscular swimming. If stimulated, they only exhibited disrupted muscular wiggling or twitching movements. In addition, their responsiveness to mechanical stimulation was reduced, with almost half of the larvae showing only brief twitches or no reaction at all. For the late RA treatment, at $24 \mathrm{hpf}$, all larvae were capable of muscular swimming. Yet, about half of them reacted in a reduced fashion or not at all when touched, while the other half would swim away normally (response A). Taken together, these data show that alteration of RA signaling levels perturbs both motorand mechanosensory functions in amphioxus larvae. Notably, the latter were more severely affected, if RA signaling levels were manipulated at late developmental stages (24 hpf).

\section{Manipulation of retinoic acid signaling levels during development negatively impacts chemosensory functions in amphioxus larvae}

In vertebrates, GLU is the most abundant excitatory neurotransmitter and contributes to most nervous system functions [71]. Therefore, the swimming patterns of amphioxus larvae were tracked and analyzed under normal conditions as well as in response to GLU exposure as a chemical stimulus (Fig. 2a; Supplementary file 2).

In the absence of exogenous GLU, control larvae swam via ciliary movement and only rarely exhibited short spontaneous phases of muscular swimming (Fig. 
$2 a, b)$. Pharmacological treatments had no effect on the occurrence of spontaneous phases of muscular swimming under these conditions. However, larvae treated with RA at 6 hpf showed an impaired muscular swimming motion that was observed slightly less often than in the control (Fig. 2b). The trajectories of control larvae mostly followed straight lines or relatively wide angles and were occasionally interrupted by periods of circling/spiraling (Fig. 2a,c). This circling/spiraling behavior was more common among larvae treated with BMS493 at either 6 or 24 hpf, but less common among larvae treated with RA at 6 hpf (Fig. 2a,c).

\section{Fig.2}

Effects of retinoic acid (RA) signaling on glutamate chemoreception in amphioxus larvae. a Experimental setup. Starting from treatment time points (t) at 6 or $24 \mathrm{hpf}$ (hours post fertilization), embryos were exposed to DMSO (Control), the RA receptor antagonist BMS493 or all-trans RA. Upon reaching the $48 \mathrm{hpf}$ stage, larvae from each treatment condition were transferred into separate Petri dishes (black circles) containing $2.5 \mathrm{ml}$ of artificial seawater. Next, a block of low-melting agarose (grey squares), which had either been dissolved in artificial seawater (no glutamate) or in artificial seawater containing $0.1 \mathrm{M} \mathrm{L-glutamate,} \mathrm{was} \mathrm{placed} \mathrm{into} \mathrm{each} \mathrm{petri} \mathrm{dish.}$ Reactions of the animals were filmed for 12 min. For subsequent analyses, only larvae that passed the agarose block within a $1 \mathrm{~cm}$ radius and within 0.5 to $10 \mathrm{~min}$ after introduction of the agarose block were considered. Colored lines depict the trajectories of 5 randomly selected larvae. Dotted lines and fat lines respectively represent slow ciliary and fast muscular swimming. Arrowheads indicate the direction of the swimming movement. b Muscular swimming events were counted for $n=50$ larvae per treatment condition. In the pie charts, the size of an individual slice corresponds to the percentage of animals that exhibited a given number of swimming events (i.e. $0=$ no muscular swimming, $1=$ a single event of muscular swimming, 2+ = two or more events of muscular swimming). $\mathbf{c}$ Swimming patterns were assessed and counted for $n=50$ animals per treatment condition. Three types of swimming patterns were distinguished: $A=$ swimming in straight lines or wide angles, $B=$ swimming in straight lines or wide angles plus periods of circling/spiraling, and $C=$ circling/spiraling for the entire observation period. In the pie charts, the size of an 
individual slice indicates the percentage of animals exhibiting a given swimming pattern.

In the presence of an agarose block containing L-GLU, most control larvae used muscular swimming to quickly move away from the L-GLU source, whenever they came too close to it (Fig. 2a). Thus, L-GLU exposure increased the occurrence of muscular swimming phases (Fig. 2b), but slightly decreased periods of circling/spiraling (Fig. 2c). Most pharmacological treatments did not affect the ability of amphioxus larvae to react to L-GLU exposure with increased muscular swimming (Fig. 2a,b). Only larvae treated with BMS493 at $24 \mathrm{hpf}$ did not react with a clear increase in muscular swimming when exposed to exogenous L-GLU (Fig. 2c). Instead, the circling/spiraling behavior that was already increased under normal conditions in larvae treated with BMS493 at either 6 or $24 \mathrm{hpf}$ became even more intense (Fig. 2c; Additional file 3: Figure S1).

Accordingly, treatments with BMS493 or RA biased ciliary swimming in amphioxus larvae towards or against circling/spiraling, respectively. Furthermore, late BMS493 treatments, starting from $24 \mathrm{hpf}$, clearly reduced larval responsiveness to LGLU as a chemical stimulus. In sum, altering RA signaling levels during amphioxus development negatively impacts larval responsiveness to both mechanical and chemical stimulation, suggesting that RA signaling is required for establishing a functional PNS.

\section{Roles of retinoic acid signaling during differentiation of the amphioxus peripheral nervous system}

Since our data indicate that RA signaling contributes to the acquisition of sensory functions in amphioxus larvae, we next wanted to determine whether it also affects PNS formation. To this end, we first documented the development of ESNs, using immunohistochemistry against the neuronal marker AT and the neurotransmitter GLU. 
At all developmental stages investigated, AT immunoreactivity (AT-ir) was present in numerous cilia of both the ectoderm and endoderm. By $15 \mathrm{hpf}$ (early neurula stage), GLU immunoreactivity (GLU-ir) and AT-ir became visible in the area of the neuropore (Fig. 3a). In late neurulae, at $24 \mathrm{hpf}$, this signal was expanded throughout the entire neural tube, including the chordoneural hinge (Fig. 3b). By 30 hpf, ESNs differentiated along the ventro-lateral ectoderm of the early larva, with each ESN projecting a single axon into the neural tube (Fig. 3c). GLU-ir was weak and confined to the somata of these early ESNs, suggesting that they were not yet fully matured. By $36 \mathrm{hpf}$, however, the amphioxus feeding larva possessed on average 42 ESNs $(\sigma=7,1 ; n=10)$, all of which displayed strong GLU-ir within both their somata and neurites (Fig. 3d). Thus, GLU neurotransmission was most likely functional at this stage. Along the anterior-posterior axis of the animals, ESNs were segmentally arranged into groups of one to four cells that generally projected their axons into the same spot within the neural tube. These projections were evenly spaced, in a manner correlated with the somite borders, indicating a corresponding segmentation of the amphioxus CNS, PNS, and mesoderm [50]. Although no external structuring is visible along the amphioxus neural tube, apart from a small bulbous cerebral vesicle [45], numerous genes are expressed in iterated segmental patterns associated with the position of the underlying somites [72]. Resembling the situation in vertebrates, the hindbrain-like domain of the amphioxus neural tube thus appears to be subdivided into a series of neuromeres that are more or less aligned with the somites and receive sensory inputs from a corresponding series of ESNs.

Most of the observed ESNs extended fine neurites across the surface of the ectoderm. In addition, several ESNs exhibited a finger-shaped element at their apex (Fig. 3e,f,g), indicative of an elongated microvilli collar, which is characteristic of putative chemosensory type II ESNs (Fig. 3e,f,g, arrowhead in f) [45, 73]. However, so far, type II ESNs have only been described in much later larval stages and as secondary sensory neurons, which possess multiple short basal processes [45, 73]. In contrast, our type II-like ESNs are primary sensory neurons with a single axon that enters the nerve cord. In late larvae, between 48 and $108 \mathrm{hpf}$, the number of ESNs increased to between 70 and 90 and a conspicuously high density of ESNs became apparent in the mid-region of the larval trunk ectoderm (Fig. 3h,i,j, dotted boxes). Contrary to early larvae, late larvae also possessed GLU-negative ESNs (Fig. 3i, 
arrowheads), with the majority of GLU-ir ESNs being located in the mid-trunk ectoderm. Besides ESNs, GLU-ir cells were further found around the orifices of late larvae, such as the pre-oral pit, the mouth, the gill slit, and the anus. However, these cells did not project processes into the CNS and were thus not considered as ESNs. Instead, some of them might belong to an independent visceral nervous system that innervates oral structures in the amphioxus larva [32, 74].

\section{Fig.3}

Development of ectodermal sensory neurons (ESNs) in amphioxus. All animals were stained with an antibody against glutamate (GLU, green), an antibody against acetylated tubulin (AT, red), and the nucleic acid dye Hoechst (blue). a Dorsal view and $\mathbf{b}$-j lateral views of amphioxus larvae at different developmental stages, from 15 to $108 \mathrm{hpf}$ (hours post fertilization). Anterior ends are directed towards the right. e-g Close-ups featuring a group of ESNs in an amphioxus larva at $36 \mathrm{hpf}$. e,f Single, color-inverted, fluorescent signal for AT-ir and and GLU-ir respectively. Arrowhead in f indicates the finger-shaped apex and fine dendrites of a type II ESN. h-j Dotted boxes indicate densely clustered ESNs in the mid-trunk ectoderm of late amphioxus larvae. Arrowheads in i indicate AT-positive, but GLU-negative, ESNs. Abbreviations: ch, cordoneural hinge; np, neuropore. Scale bar in a also applies to $\mathbf{b}-\mathbf{d}$, scale bar in e also applies to $\mathbf{f , g}$ and scale bar in $\mathbf{h}$ also applies to $\mathbf{i , j}$.

We next assessed how pharmacological manipulation of RA signaling levels at 6, 12 or $24 \mathrm{hpf}$ affects ESN formation. In order to quantify and compare the distribution of GLU/AT-ir ESNs, amphioxus larvae at $36 \mathrm{hpf}$ were subdivided along their anterior-posterior axis into three territories of equal size. In control animals, the anterior part comprised on average 15, the middle part 11, and the posterior part 16 ESNs (Fig. 4a,a',e,e',i). Animals that were treated with BMS493 at 6 hpf did not exhibit any significant changes in the number and distribution of their ESNs. In contrast, animals that were treated with RA at $6 \mathrm{hpf}$ possessed significantly more ESNs in their anterior and middle parts, but less in their posterior part (Fig. 
$\left.4 b, b^{\prime}, f, f^{\prime}, i\right)$. The overall amount of ESNs in these animals did, however, not change significantly. Accordingly, early alterations of RA signaling levels only affected the anterior-posterior position of ESNs. Later treatments, at $12 \mathrm{hpf}$, on the other hand, significantly increased the overall number of ESNs, either in middle and posterior parts for BMS493 treatments or in anterior and middle parts for RA treatments (Fig. $\left.4 c, c^{\prime}, g, g^{\prime}, i\right)$. Treatments that began even later, at $24 \mathrm{hpf}$, caused an even greater increase in the overall amount of ESNs (Fig. 4d,d',h,h',i). Notably, a higher number of ESNs was again observed in middle and posterior parts for BMS493 treatments at 24 hpf, and all along the anterior-posterior axis for RA treatments at $24 \mathrm{hpf}$. To corroborate these findings, we also investigated how manipulation of RA signaling levels influences the expression of vglut, a marker gene of glutamatergic neurons. These experiments led to results consistent with those obtained by assaying the distribution of GLU/AT-ir ESNs (Additional file 4: Figure S2). Accordingly, our data demonstrate that RA signaling regulates ESN formation in a stage- and tissuedependent manner.

Fig.4

Effects of retinoic acid (RA) signaling on the development of ectodermal sensory neurons (ESNs) in amphioxus. a-d' Larvae at $36 \mathrm{hpf}$ (hours post fertilization) were stained for glutamate-immunoreactivity (GLU-ir, green), acetylated tubulin-ir (AT-ir, red), and with the nucleic acid dye Hoechst (blue). e-h' Schematic drawings of the animals shown in a-d'. Outlines of the animals and their pre-oral pits are depicted in grey, the gut in bright yellow, and neural projections in red. ESNs are depicted as circles with a red outline that are filled with either green or yellow color to represent the presence or absence of GLU-ir respectively. Animals had been treated with either DMSO (Control, a,a',e,e'), with the RA receptor (RAR) antagonist BMS493 (b-d,f-h), or with all-trans RA (b'-d',f'-h'), starting from different treatment time points (t), at either 6 or $24 \mathrm{hpf}$, as indicated. i Statistical analyses of the observed effects of RA signaling on ESN formation. Colored bars depict the average number of ESNs in the whole ectoderm (All), or in specific ectodermal sections along the anterior-posterior axis. The image below the first diagram shows Hoechst labeling (grey) in a larva at $36 \mathrm{hpf}$, with dotted lines marking the sections of the ectoderm (Posterior, Middle, 
Anterior), in which ESNs were counted. Green bars stand for control animals, blue bars for animals treated with BMS493, and red bars for animals treated with all-trans RA. For each diagram, treatment time points $(\mathrm{t})$, at 6 or $24 \mathrm{hpf}$, are given in the upper right corner and the number $(n)$ of animals counted per condition is indicated at the base of the first three colored bars. Error bars depict the standard deviation ( $\sigma)$. An asterisk $\left(^{*}\right)$ above an error bar indicates that the difference between this condition and the corresponding control is statistically significant. The scale bar in a also applies to $\mathbf{b}-\mathbf{d}, \mathbf{a}^{\prime}-\mathbf{d}^{\prime}$, and the scale bar in e also applies to f-h,e'-h'.

\section{Roles of retinoic acid signaling in ectodermal cell proliferation and apoptosis}

In an effort to understand how RA signaling modulates ESN development in amphioxus, we next tested how altering RA signaling levels at either 6 or $24 \mathrm{hpf}$ influences ectodermal cell proliferation and apoptosis.

Using the 5-ethynyl-2'-deoxyuridine (EdU) labeling technique, we thus assessed cell proliferation during a specific developmental timeframe. In amphioxus larvae exposed to EdU from 24 to $28 \mathrm{hpf}$ and fixed at $36 \mathrm{hpf}$, most EdU-positive nuclei were observed in the gut, but were also detectable in the cerebral vesicle, in the posterior neural tube, and dispersed across the ectoderm (Fig. 5). The labeled nuclei exhibited different signal intensities, due to signal dilution, which occurs in cells that kept dividing after the $4 \mathrm{~h}$ EdU pulse. Accordingly, cells with strongly labeled nuclei proliferated during the EdU pulse, whereas cells with weakly labeled nuclei underwent additional divisions after the EdU pulse. Most ectodermal nuclei showed weak EdU labeling, indicating continuous proliferative activity after the EdU pulse. On average $49 \mathrm{EdU}$-positive nuclei were detected within the ectoderm of control larvae $(\sigma=10 ; n=15)$ (Fig. 5a,b,b',e). The majority of these nuclei were located in the anterior and tail region of the larvae, while only very few were found in the mid-trunk region. RA treatments at 6 and $24 \mathrm{hpf}$ had no significant effect on the number or distribution of ectodermal EdU-positive nuclei (Fig. 5c',d',e). In contrast, treatments with BMS493 at both 6 and $24 \mathrm{hpf}$ led to a significant increase in ectodermal EdU-positive nuclei, especially in the anterior part of the larvae (Fig. 5c,d,e). These results suggest that 
RA signaling functions during amphioxus development to limit cell proliferation in the larval ectoderm.

\section{Fig.5}

Effects of retinoic acid (RA) signaling on cell proliferation in the ectoderm of amphioxus larvae. a-d' Animals at 36 hpf (hours post fertilization) are shown in lateral view with their anterior ends directed towards the right. a Larva that was stained for 5-ethynyl-2'-deoxyuridine (EdU, yellow), for glutamate-immunoreactivity (GLU-ir, green), for acetylated tubulin-ir (AT-ir, red), and with the nucleic acid dye Hoechst (blue). b-d' Images showing only the color-inverted fluorescent signal for EdU (grey). a-b' Control animals treated with DMSO. c,d Animals treated with the RA receptor (RAR) antagonist BMS493. c',d' Animals treated with all-trans RA. The treatment time points $(\mathrm{t})$, at either 6 or $24 \mathrm{hpf}$, are given in the upper right corner of each image. e Statistical analyses of the observed effects of RA signaling on ectodermal cell proliferation. Colored bars depict the average number of EdU-positive nuclei present in the whole ectoderm (All) or in specific ectodermal sections along the anterior-posterior axis. The image below the first diagram shows Hoechst labeling (grey) in a larva at $36 \mathrm{hpf}$, with dotted lines marking the sections of the ectoderm (Posterior, Middle, Anterior), in which EdU-positive nuclei were counted. Green, blue, and red bars stand respectively for control animals, for animals treated with BMS493, and for animals treated with all-trans RA. For each diagram, treatment time points $(\mathrm{t})$, at either 6 or $24 \mathrm{hpf}$, are given in the upper right corner, and the number ( $\mathrm{n}$ ) of animals that were counted per condition is indicated at the base of the first three colored bars. Error bars depict the standard deviation $(\sigma)$. Asterisks $\left(^{*}\right)$ above error bars indicate that the difference between this condition and the corresponding control is statistically significant. The scale bar in $\mathbf{b}$ also applies to $\mathbf{c}, \mathbf{d}, \mathbf{b}^{\prime} \mathbf{-}-\mathbf{d}$ '.

Terminal deoxynucleotidyl transferase dUTP nick end labeling (TUNEL) assays can be used to reveal DNA fragmentation, which occurs during the late stages of apoptosis. In amphioxus larvae at $60 \mathrm{hpf}$, apoptotic cells were present at 
the rostral end of the animals, in the vicinity of the mouth and gill slit, and in individual patches that were dispersed across the ectoderm (Fig. 6a). BMS493 treatments at 6 hpf resulted in an increase of apoptotic cells within the general ectoderm of amphioxus larvae that was not seen for late BMS493 treatments, at 24 hpf (Fig. $6 \mathrm{~b}, \mathrm{c})$. Conversely, animals treated with RA at 6 hpf showed no apparent changes in the amount or distribution of apoptotic cells within their ectoderm (Fig. 6b'). If RA treatments began later, at $24 \mathrm{hpf}$, fewer apoptotic cells were visible in the general ectoderm, with most apoptotic cells occupying the area of the anterior pharynx (Fig. $\left.6 c^{\prime}\right)$. Thus, RA signaling seems to promote ectodermal cell survival in amphioxus larvae. In sum, these findings show that RA signaling reduces the proliferation and promotes the survival of ectodermal cells in amphioxus larvae. However, it was not possible to establish a clear correlation between these functions and the observed RA signaling-dependent regulation of ESN formation.

\section{Fig. 6}

Effects of retinoic acid (RA) signaling on apoptosis in amphioxus larvae. Animals at 60 hpf (hours post fertilization) were stained with a terminal deoxynucleotidyl transferase dUTP nick end labeling (TUNEL) kit for apoptotic cells (yellow) and with the nucleic acid dye Hoechst (blue). All larvae are shown in lateral view with their anterior ends directed towards the right. a Control animal that was treated at $6 \mathrm{hpf}$ with DMSO. b,c Animals that were treated with the RA receptor (RAR) antagonist BMS493 at two different developmental stages, 6 or 24 hpf. b',c' Animals that were treated with all-trans RA at two different developmental stages, 6 or $24 \mathrm{hpf}$. The treatment time point $(t)$ is given in the upper right corner of each image. Scale bar in (A) applies to all images.

\section{Formation of the amphioxus peripheral nervous system from at least two distinct populations of ectodermal sensory neuron progenitors}

In order to better understand ESN formation in amphioxus, we decided to compare the number and distribution of ESNPs expressing different neural marker 
genes. Since the developmental expression of hu/elav, t/x, and soxb1c in $B$. laceolatum largely corresponded to that previously described in other amphioxus species [49-52, 75], the following sections will focus exclusively on the expression of these neural marker genes in B. lanceolatum ESNPs.

Expression of the neuron-specific RNA binding protein hu/elav became visible in individual ESNPs at about $18 \mathrm{hpf}$ (Additional file 5: Figure S3a,a',b,b'). Initially, these hu/elav-expressing ESNPs were located in the ventral ectoderm of the midneurula stage embryo. As development proceeded, the number of hu/elav-positive ESNPs increased and their location changed from ventral to lateral ectoderm (Additional file 5: Figure S3c,c',d,d',e,e'). During early larval stages, between 30 and $36 \mathrm{hpf}$, hu/elav-expressing ESNPs were evenly dispersed along the anterior-posterior axis, excepting a conspicuously dense accumulation in the mid-region of the trunk (Additional file 5: Figure S3d,d',e,e'). Notably, this is consistent with the abovedescribed particularly high density of GLU/AT-ir ESNs in the mid-trunk area of later larval stages, between 48 and 108 hpf (Fig. 3h,i,j).

The nuclear transcription factor $t / x$ was already expressed throughout the ventral ectoderm of early amphioxus neurulae, at 15 and $18 \mathrm{hpf}$ (Additional file 6: Figure S4a,a',b,b'). By $24 \mathrm{hpf}$, this ectodermal t/x expression became confined to individual ESNPs (Additional file 6: Figure S4c,c'). Importantly, however, less t/xexpressing ESNPs than hu/elav-expressing ESNPs were observed (Additional file 6: Figure S4c,c',d,d',e,e'). As for the majority of hu/elav-expressing ESNPs, t/xexpressing ESNPs were evenly dispersed along the lateral ectoderm of amphioxus larvae at 30 and 36 hpf (Additional file 6: Figure S4d,d',e,e'). But contrary to hu/elavexpressing ESNPs, no accumulation of $t / x$-expressing ESNPs was observed in the mid-trunk region of the larvae (Additional file 6: Figure S4d,d',e,e'). Taken together, these data indicate that $t / x$ is only expressed in a subset of amphioxus ESNPs, because in the mid-trunk area of late neurulae and larvae a dense accumulation of ESNPs can be detected by hu/elav but not by $t / x$ expression.

The HMG-box transcription factor soxb1c only became expressed in a few ESNPs by the late neurula stage, at $24 \mathrm{hpf}$ (Additional file 7: Figure S5a,a',b,b',c,c'). Between 30 and $36 \mathrm{hpf}$, the number of soxb1c-expressing ESNPs increased to four to seven (Additional file 7: Figure S5d,d',e,e'). Notably, all of them were localized in 
the mid-trunk region, where a particularly dense accumulation of ESNPs was labeled by hu/elav but not by $t / x$ expression. Since $t / x$-expressing ESNPs are positioned slightly more ventral within the lateral ectoderm than soxb1c-expressing ESNPs, these two genes likely mark distinct populations of ESNPs. In addition, our data indicate a link between the soxb1c-expressing ESNPs and the conspicuous accumulation of GLU/AT-ir ESNs in the mid-trunk ectoderm of amphioxus larvae.

To further scrutinize the respective distribution of hu/elav-, t/x-, and soxb1cexpressing ESNPs, we next co-localized the expression of each of these genes with GLU- and AT-ir, which labels mature ESNs in amphioxus larvae at $36 \mathrm{hpf}$. Expression of hu/elav was visible in all differentiated ESNs as well as in undifferentiated ESNPs in the mid-trunk region of the larva (Fig. 7a,a'). In contrast, $t / x$ expression was only observed in a subset of differentiated ESNs (Fig. 7b,b'), and soxb1c was exclusively expressed in undifferentiated ESNPs that were concentrated in the mid-trunk region of the larva (Fig. 7c,c'). The larval amphioxus PNS thus appears to develop from at least two distinct populations of ESNPs: one that arises during early neurula stages (15 to $18 \mathrm{hpf}$ ) from the ventral ectoderm and expresses $t / x$ and another one that arises during later neurula stages (18 to $24 \mathrm{hpf}$ ) in the lateral ectoderm and expresses soxb1c. Our results further suggest that the t/x-expressing ESNPs differentiate promptly into ESNs, while the soxb1c-expressing population is maintained during later development to supply the elongating larvae with additional GLU-ir ESNs (Fig. 3h,i,j).

\section{Fig.7}

Co-localization of neural marker gene expression with ectodermal sensory neurons (ESNs) in amphioxus larvae. All animals are shown in lateral view with their anterior end directed toward the right. a,a' hu/elav, b,b' $t / x$, and $\mathbf{c}, \mathbf{c}^{\prime}$ soxb1c gene expression (yellow) was detected in amphioxus larvae at $36 \mathrm{hpf}$ (hours post fertilization). ESNs were labeled by immunostaining against glutamate (GLU-ir, green) and acetylated tubulin (AT-ir, red). Nuclei were labeled with the nucleic acid dye Hoechst (blue). The white boxes in a-c indicate the position of the respective close-ups shown in a'-c'. Close-ups were generated from confocal z-stacks that only include the lateral 
ectoderm on one side of the larvae. Arrows in a' indicate differentiated ESNs expressing hu/elav and the arrowhead an undifferentiated ESN progenitor (ESNP) expressing hu/elav. The arrow in b' highlights a differentiated ESN expressing $t / x$ and the arrowhead a differentiated ESN that does not express t/x. Arrows in c' mark differentiated ESNs that do not express soxb1c and the arrowheads undifferentiated ESNPs expressing soxb1c. The scale bar in a also applies to $\mathbf{b}, \mathbf{c}$, and the scale bar in $\mathbf{a}^{\prime}$ also applies to $\mathbf{b}^{\prime}, \mathbf{c}^{\prime}$.

\section{Roles of retinoic acid signaling during ectodermal sensory neuron progenitor development}

Finally, we analyzed how pharmacological manipulation of RA signaling levels at either 6 or $24 \mathrm{hpf}$ affects the formation of ESNPs expressing hu/elav, t/x, and soxb1c in amphioxus larvae at $30 \mathrm{hpf}$. To this end, the larvae were divided into three sections of equal size along their anterior-posterior axis (i.e. into an anterior, a middle, and a posterior section), and the number of ESNPs per section was counted (Fig. 11). Of note, ESNPs were only counted in the lateral ectoderm on one side of developing amphioxus larvae, whereas GLU-/AT-ir ESNs were counted on both sides of the larvae.

Control larvae possessed on average 24.5 hu/elav-expressing ESNPs ( $\sigma=3.4$; $\mathrm{n}=12)$ at $30 \mathrm{hpf:} 6.8$ in the anterior $(\sigma=1.3), 11.1$ in the mid-trunk $(\sigma=1.7)$, and 6.7 in the posterior region $(\sigma=1.4)$ (Fig. 11a). In animals that were treated with the RAR antagonist BMS493 at $6 \mathrm{hpf}$, hu/elav-expressing ESNPs appeared to be slightly shifted towards more posterior levels (Fig. 8a,a',b,b',f,f',g,g'), but no statistically significant changes in their number and distribution could be detected (Fig. 11a). In contrast, RA treatment at $6 \mathrm{hpf}$ strongly increased the overall number of hu/elavexpressing ESNPs to $38.9(\sigma=8.9 ; n=10)$. Most of these additional ESNPs were observed in mid-trunk and anterior regions of the larvae, which respectively contained $16.6(\sigma=6.0)$ and $15.6(\sigma=2.7)$ ESNPs (Fig. 8a,a',c,c',f,f',h,h', Figure 11a). Later treatments, at $24 \mathrm{hpf}$, also increased the overall number of hu/elav-expressing ESNPs, to $27.3(\sigma=2.1 ; n=8)$ for the BMS493 treatment and to $30.7(\sigma=2.7 ; n=10)$ for the RA treatment (Fig. 11a). These additional ESNPs were chiefly located in the 
posterior region of BMS493-treated animals (Fig. 8a,a',d,d',f,',i,i'), which thus contained 9.6 ESNPs $(\sigma=1.1)$, or in the anterior and posterior regions of RA-treated animals (Fig. 8a,a',e,e',f,f',j,'), which respectively contained $10.0(\sigma=1.6)$ and 9.0 ESNPs $(\sigma=0.7)$ (Fig. 11a). These results are largely consistent with those obtained for GLU- and AT-ir ESNs (Fig. 4). Furthermore, similar effects were observed for vglut-expressing cells in the ectoderm of amphioxus larvae (Additional file 4: Figure S2).

\section{Fig. 8}

Effects of retinoic acid (RA) signaling on development of hu/elav-expressing cells in amphioxus. a-e' Larvae at $30 \mathrm{hpf}$ (hours post fertilization) and f-j' larvae at $36 \mathrm{hpf}$ are shown in lateral view (a-j) and dorsal view (a'-j'). a,a',f,f' Control animals treated with DMSO at 6 hpf. Dotted lines indicate the mid-trunk area, where a particularly high density of ectodermal sensory neuron progenitors (ESNPs) is observed (compare with Additional file 5: Figure S3). $\mathbf{b}, \mathbf{b}^{\prime}, \mathbf{d}, \mathbf{d}^{\prime}, \mathbf{g}, \mathbf{g}^{\prime}, \mathbf{i}, \mathbf{i} \mathbf{i}^{\prime}$ Animals treated with the RA receptor (RAR) antagonist BMS493 at two different developmental stages, 6 or 24 hpf. c, c',e,e','h,h',j,j' Animals treated with all-trans RA at two different developmental stages, 6 or $24 \mathrm{hpf}$. The treatment time point (t), 6 or $24 \mathrm{hpf}$, is indicated in the upper right corner of each image. Scale bars are $50 \mu \mathrm{m}$. The scale bar in a also applies to $\mathbf{a}^{\prime}, \mathbf{b}-\mathbf{e}^{\prime}$, and the scale bar in $\mathbf{f}$ also applies to $\mathbf{f}^{\prime}, \mathbf{g}-\mathbf{j}^{\prime}$.

In contrast to hu/elav-expressing ESNPs, t/x-expressing ESNPs were largely unaffected by treatments with BMS493 or RA (Fig. 9, Figure 11b). Only early RA treatments at $6 \mathrm{hpf}$ slightly shifted the t/x-expressing ESNPs anteriorly, thus eliminating them from the most posterior regions of the amphioxus tail ectoderm (Fig. 9a,a',c,c',f,f',h,h', Figure 11b). Therefore, tlx-expressing ESNPs do not appear to majorly contribute to the observed effects of altered RA signaling levels on ESN formation. 


\section{Fig.9}

Effects of retinoic acid (RA) signaling on the development of $t / x$-expressing cells in amphioxus. a-e' Larvae at $30 \mathrm{hpf}$ (hours post fertilization) and f-j' larvae at $36 \mathrm{hpf}$ are shown in lateral view (a-j) and dorsal view (a'-j'). $\mathbf{a}, \mathbf{a}^{\prime}, \mathbf{f}, \mathbf{f}^{\prime}$ Control animals treated, at 6 hpf, with DMSO. $\mathbf{b}, \mathbf{b}^{\prime}, \mathbf{d}, \mathbf{d}^{\prime}, \mathbf{g}, \mathbf{g}^{\prime}, \mathbf{i}, \mathbf{i}$ ' Animals treated with the RA receptor (RAR) antagonist BMS493 at two different developmental stages, 6 or $24 \mathrm{hpf}$. $\mathbf{c}, \mathbf{c}^{\prime}, \mathbf{e}, \mathbf{e} \mathbf{e}^{\prime}, \mathbf{h}, \mathbf{h}^{\prime}, \mathbf{j}, \mathbf{j}$ ' Animals treated with all-trans RA at two different developmental stages, 6 or $24 \mathrm{hpf}$. The treatment time point (t), 6 or $24 \mathrm{hpf}$, is indicated in the right upper corner of each image. Scale bars are $50 \mu \mathrm{m}$. The scale bar in a also applies to $\mathbf{a}^{\prime}, \mathbf{b}-\mathbf{e}^{\prime}$, and the scale bar in $\mathbf{f}$ also applies to $\mathbf{f}^{\prime}, \mathbf{g}-\mathbf{j}^{\prime}$.

In control larvae, a small population of 4.5 soxb1c-expressing ESNPs ( $\sigma=1.1$; $\mathrm{n}=12$ ) can be observed in the mid-trunk ectoderm at $30 \mathrm{hpf}$ (Fig. 11c). This population was shifted towards more posterior levels by BMS493 treatments at $6 \mathrm{hpf}$, so that the mid-trunk region lost on average 1.7 ESNPs $(\sigma=0.7 ; n=12)$, while the posterior region gained 1.7 ESNPs ( $\sigma=0.6)$ (Fig. 10a,a',b,b',f,f',g,g', Fig. 11c). In contrast, RA treatment at $6 \mathrm{hpf}$ increased the overall number of soxb1c-expressing ESNPs to 16.1 ( $\sigma=2.7 ; n=12)$, with additional ESNPs mainly occupying the mid-trunk and anterior regions (Fig. 10a,a',c,c',f,',h,h', Fig. 11c). Late BMS493 treatments, at $24 \mathrm{hpf}$, also increased the overall number of soxb1c-expressing ESNPs to 7.3 $(\sigma=1.6 ; n=10)$, but additional soxb1c-expressing ESNPs were exclusively localized in the posterior region, which thus contained 2.4 ESNPs ( $\sigma=0.8$ ) (Fig. 10a,a',d, d',f,f',i,i', Fig. 11c). Late RA treatments, at $24 \mathrm{hpf}$, increased the amount of soxb1c-expressing ESNPs mainly in the mid-trunk ectoderm, but also in anterior and posterior regions (Fig. 10a,a',e,e',f,f',j,j', Fig. 11c). These results are in agreement with the effects of RA signaling alterations on the development of hu/elav-expressing ESNPs (Fig. 8, Fig. 11a) and GLU-/AT-ir ESNs (Fig. 4). In sum, our data thus show that RA signaling influences the number and distribution of amphioxus ESNs, mainly by acting on late soxb1c-expressing ESNPs, which form a neurogenic niche in the mid-trunk ectoderm of amphioxus larvae. 


\section{Fig.10}

Effects of retinoic acid (RA) signaling on the development of soxb1c-expressing cells in amphioxus. a-e' Larvae at $30 \mathrm{hpf}$ (hours post fertilization) and f-j' larvae at $36 \mathrm{hpf}$ are shown in lateral view (a-j) and dorsal view (a'-j'). All images are focused only on soxb1c-expressing cells in the ectoderm. a,a',f,f' Control animals treated at $6 \mathrm{hpf}$ with DMSO. b,b',d,d',g,g',i,i,' Animals treated with the RA receptor (RAR) antagonist BMS493 at two different developmental stages, 6 or 24 hpf. c,c',e,e',h,h',j,j' Animals treated with all-trans RA at two different developmental stages, 6 or $24 \mathrm{hpf}$. The treatment time point $(\mathrm{t}), 6$ or $24 \mathrm{hpf}$, is indicated in the right upper corner of each image. a-e' Dotted lines mark the ectodermal domain that contains soxb1cexpressing ectodermal sensory neuron progenitors (ESNPs) (compare with Additional file 7: Figure S5). d,d' Arrowheads mark soxb1c-expressing ESNPs that are induced specifically in the tail ectoderm of early larvae, which had been treated with BMS493 at $24 \mathrm{hpf}$. Scale bars are $50 \mu \mathrm{m}$. The scale bar in a also applies to $\mathbf{a}^{\mathbf{\prime}}, \mathbf{b}-$ $\mathbf{e}^{\prime}$, and the scale bar in $\mathbf{f}$ also applies to $\mathbf{f}^{\prime}, \mathbf{g}-\mathbf{j}$ '.

\section{Fig.11}

Statistical analyses of retinoic acid (RA) signaling effects on amphioxus ectodermal sensory neuron progenitors (ESNPs). The number and anterior-posterior distribution of a hu/elav-, b t/x-, and c soxb1c-expressing ESNPs was assessed in larvae at 30 hpf (hours post fertilization). ESNPs were only counted in the lateral ectoderm on one side of the animals. Colored bars depict the average number of ESNPs either in the entire lateral ectoderm (All) or in a specific ectodermal section along the anteriorposterior axis (Posterior, Middle, Anterior). Green bars stand for control animals, blue bars stand for animals treated with the RA receptor (RAR) antagonist BMS493, and red bars stand for animals treated with all-trans RA. Treatments began either at 6 (solid colored bars) or 24 hpf (striped colored bars). The number ( $\mathrm{n}$ ) of animals that were counted for each condition is indicated at the base of the first five colored bars. The error bars depict the standard deviation $(\sigma)$, and an asterisk $\left(^{*}\right)$ above an error bar indicates that the difference between this condition and the corresponding control is statistically significant. 


\section{Discussion}

In vertebrates, RA signaling influences the development of neural crest and neurogenic placode derivatives, which give rise to portions of the PNS [17], and thus also contributes to the establishment of neurobehavior requiring PNS input [1-5]. However, information about these RA signaling functions is still fragmentary and it is currently unclear whether they are conserved among chordates. In this study, we hence assessed how RA signaling contributes to the ontogeny of sensory functions and of the PNS in the cephalochordate amphioxus, a good proxy for the ancestral chordate condition [47, 73]. In amphioxus, RA signaling commences at mid-gastrula stages, when the single amphioxus rar gene becomes expressed throughout the mesoderm and weakly throughout the ectoderm [41]. In addition, six aldh1 duplicates, which encode potential RA synthesis enzymes, become expressed in posterior mesendodermal domains of the embryo [76]. RA degrading enzymes of amphioxus include CYP26-1, CYP26-2, and CYP26-3 [77]. During development, cyp26-2 is the predominantly expressed gene that is first detectable around the blastopore of mid-gastrula stages [77]. By the mid-neurula stage, cyp26-2 expression is present in the two anterior-most somites, in the anterior- and posterior-most ectoderm as well as in the anterior- and posterior-most endoderm [77]. Thus, RA signaling in the amphioxus embryo most likely involves a posterior RA source, from where the morphogen diffuses, creating a posterior-high signaling gradient that is degraded in distinct anterior and posterior domains. A similar RA gradient has been visualized in zebrafish [78], supporting the notion that RA signaling levels play important context-dependent roles during chordate ontogeny.

Accordingly, we first tested whether altering RA signaling levels during amphioxus embryogenesis affects sensory functions. Our results show that both inhibiting and increasing RA signaling levels has a negative impact on the ability of amphioxus larvae to react to external stimuli. Notably, responsiveness to mechanical as well as chemical stimulation was most severely impaired, if RA signaling was inhibited starting from late neurula (24 hpf) rather than earlier blastula (6 hpf) stages. In contrast, early exposure to excess RA caused mainly motor impairments. These findings strongly suggest that RA signaling contributes to different aspects of 
amphioxus PNS development in a stage-dependent manner. Therefore, we next investigated how RA signaling influences differentiation of the amphioxus PNS.

Using antibodies against AT and GLU as markers, we were able to detect a large set of about 40 ESNs that arises more or less simultaneously at about $30 \mathrm{hpf}$ and is evenly distributed along the anterior-posterior axis of amphioxus larvae at 36 hpf. Manipulation of RA signaling levels during amphioxus embryogenesis significantly changed both the number and the distribution of these ESNs in a stagedependent manner. Altering endogenous RA signaling levels at early stages mostly caused minor shifts in the distribution of ESNs along the anterior-posterior axis. In contrast, later inhibition or increase of RA signaling activity led to the formation of additional ESNs in mid-trunk and posterior ectoderm or in mid-trunk and anterior ectoderm, respectively. Interestingly, the responsiveness of amphioxus larvae to external stimulation was most severely reduced by late manipulation of RA signaling levels. A potential explanation could be that the presence of excess GLU-ir ESNs causes a mismatch between excitatory and inhibitory inputs, which impairs sensory information processing. Early exposure to excess RA further caused motor impairments associated with an overall anterior shift of ESNs. Since sensory feedback is crucial for the control of motor functions [79, 80], these motor impairments might be, at least in part, due to a lack of ESNs in the tail region. Moreover, it has been reported that altering RA signaling levels during early amphioxus development affects the composition of ESN types along the anteriorposterior axis [29], which is likely to perturb the assembly of functional neuronal circuits.

In order to better understand how RA signaling controls ESN formation, we subsequently analyzed proliferation and apoptosis patterns in the ectoderm of amphioxus larvae. In the nervous system of vertebrates, RA signaling is known to simultaneously act as a permissive differentiation signal, by repressing the FGFdependent maintenance of progenitor cell function, and as an instructive signal that induces neuronal differentiation [81, 82]. Furthermore, RA signaling can determine the precise timing of stem cell differentiation by generating heritable epigenetic changes in chromatin responsiveness to both RA and other signaling pathways [83] and may exert opposite effects on cell survival and cell cycle, depending on the 
presence of specific binding proteins [84, 85]. In amphioxus, upregulation of RA signaling did not overtly alter ectodermal proliferation rates, although a slight reduction of apoptosis was observed. In contrast, downregulation of RA signaling increased proliferation rates, while apoptosis also seemed to be slightly elevated, but only for animals in which RA signals were blocked from an early stage. These results are consistent with a role for RA signaling as a positive regulator of cell differentiation and cell survival in amphioxus. However, it was not possible to correlate RA signaling-dependent differences in proliferation and apoptosis with concurrent changes in the number and distribution of ESNs.

Consequently, we next investigated the development of amphioxus ESNPs that express different neural marker genes, such as hu/elav, t/x, and soxb1c. Our data show that the hu/elav gene is most likely expressed in all ESNPs as well as in all differentiated ESNs of amphioxus embryos and larvae. During gastrula and neurula stages, the $t / x$ gene is also expressed in a large population of ESNPs that migrate from the ventral to the ventro-lateral ectoderm. However, in the amphioxus larva, t/x expression is only maintained in a subset of differentiated ESNs. In contrast, the soxb1c gene is expressed in only a small population of late ESNPs that is located at dorso-lateral levels of the mid-trunk ectoderm in amphioxus neurulae and larvae. Since the number of ESNs keeps increasing during amphioxus larval development [45] and since a particularly high density of ESNPs and ESNs can be observed in the mid-trunk ectoderm, this soxb1c-expressing ESNP population likely forms a neurogenic niche, which supplies new ESNs as the animals elongate. Consistently, soxb1 transcription factors have been shown to promote neural progenitor proliferation, while suppressing their differentiation in various taxa, including cnidarians [86], arthropods [87], echinoderms [88, 89], hemichordates [90], and vertebrates [91].

We then compared how the development of amphioxus ESNs and different ESNP populations is affected by manipulation of RA signaling levels (for an overview see Table 1). Importantly, we found that RA signaling influences the formation of late soxb1c-expressing ESNPs in a stage-dependent manner, whereas they have little effect on early $t / x$-expressing ESNPs. Our data indicate that during early neurula stages, RA signaling specifies the mid-trunk territory in which the soxb1c-expressing 
ESNP population will form, while also playing a minor role in regulating the anteriorposterior distribution of $t / x$-expressing ESNPs. At later stages, RA signaling then contributes to the restricted maintenance of soxb1c-expressing ESNPs within the mid-trunk ectoderm, thus controlling ESN production. These functions likely rely on a posterior high gradient of RA signaling: medium levels of RA in the mid-trunk ectoderm stimulate the specification of late soxb1c-expressing ESNPs and ESN production, while high levels of RA prevent this process in the tail ectoderm (Fig. 12). Consistently, the amphioxus rar is most strongly expressed in the mid-trunk ectoderm of amphioxus larvae and reacts to early alterations of RA signaling levels in a similar manner as the soxb1c-expressing ESNP population [29, 41]. Taken together, these data support a role for medium RA signaling levels in specifying and regulating a neurogenic niche in the mid-trunk ectoderm of amphioxus larvae.

\begin{tabular}{|c|c|c|c|c|c|}
\hline \multicolumn{2}{|c|}{ Neural cell population } & \multicolumn{4}{|c|}{ Pharmacological treatment effects } \\
\hline Marker & Region & t6 BMS493 & t24 BMS493 & t6 RA & t24 RA \\
\hline GLU-/AT-ir & Anterior & 0 & 0 & + & + \\
\hline GLU-/AT-ir & Middle & 0 & + & + & + \\
\hline GLU-/AT-ir & Posterior & 0 & + & - & + \\
\hline Hu/elav & Anterior & 0 & 0 & + & + \\
\hline Hu/elav & Middle & 0 & 0 & + & $+/ 0$ \\
\hline Hu/elav & Posterior & 0 & + & 0 & + \\
\hline$T / x$ & Anterior & 0 & 0 & 0 & 0 \\
\hline$T / x$ & Middle & 0 & 0 & 0 & 0 \\
\hline$T / x$ & Posterior & 0 & 0 & - & 0 \\
\hline Soxb1c & Anterior & 0 & 0 & + & $+/ 0$ \\
\hline Soxb1c & Middle & - & 0 & + & + \\
\hline Soxb1c & Posterior & + & + & 0 & + \\
\hline \multicolumn{2}{|c|}{ Sensory functions } & $\begin{array}{c}\text { Mildly } \\
\text { impaired }\end{array}$ & $\begin{array}{l}\text { Strongly } \\
\text { impaired }\end{array}$ & Impaired & Impaired \\
\hline
\end{tabular}

Table 1: Summary of the observed RA signaling effects on neural development in amphioxus. Results were obtained from immunohistochemical and gene expression analyses of ectodermal sensory neurons (ESNs) and ESN progenitors (ESNPs) 
following pharmacological treatments of developing amphioxus with the retinoic acid receptor (RAR) antagonist BMS493 or all-trans retinoic acid (RA). The developmental time point ( $\mathrm{t}$ ) of the treatments, at either 6 or $24 \mathrm{hpf}$ (hours post fertilization), is given together with the compound. The observed effects on the formation of selected neural cell populations and on sensory functions are indicated. Treatment outcomes for the different neural populations are depicted as follows: $0=$ no effect, $+=$ gain of cells, - = loss of cells. Minor effects are indicated with a "/0". Abbreviations: GLU-/ATir - glutamate/acetylated tubulin immunoreactivity.

\section{Fig.12}

Retinoic acid (RA) signaling dependence of ectodermal sensory neuron (ESN) formation in amphioxus. Schematic representation of an amphioxus larva at 30 hours post ferilization (hpf) with the anterior end directed towards the right. The central nervous system (CNS) is drawn in orange, the gut is drawn in gray, and a posteriorhigh gradient of RA signaling is indicated in magenta. ESN progenitors (ESNPs) are depicted as small ovals. Ectodermal expression of neural marker genes is shown as a black outline for hu/elav, as green filling for $t / x$, and as blue filling for soxb1c. Initially, $t / x$ is widely expressed in the ventral ectoderm, but gradually becomes restricted to individual ESNPs that migrate dorsally. During early embryogenesis, RA signaling levels mildly influence the distribution of $t / x$-expressing ESNPs. The hu/elav gene is likely expressed in all specified ESNPs. In contrast, soxb1c expression is only detected in a small population of late developing ESNPs, located at mid-trunk levels in the dorso-lateral ectoderm. The single amphioxus rar gene is also most strongly expressed in this ectodermal domain [29, 41], as indicated by the blackoutlined rhombus shown above the larva. Our data suggest that medium levels of RA signaling in the mid-trunk ectoderm contribute to specification of the soxb1cexpressing ESNP population and regulate its neurogenic activity.

Interestingly, it has previously been postulated that the source of endogenous $\mathrm{RA}$ as well as the rar expression domain move caudally, while the amphioxus larva 
elongates [92]. In this case, the ectodermal zone receiving medium levels of RA signaling, which are required for maintaining a soxb1c-expressing neurogenic niche, would also gradually be shifted towards more posterior levels. Thus, ESN production is probably matched to the posterior extension of the larval body and to the formation of new pharyngeal structures (e.g. gill slits), which also occurs in an anterior-toposterior progression and was shown to depend on RA signaling [92].

The neurogenic niche in the lateral ectoderm of amphioxus larvae shares several similarities with vertebrate placodes. For instance, the vertebrate placodal marker genes six1/2 and eya are specifically expressed in a subset of amphioxus ESNPs in the mid-trunk ectoderm that corresponds well to the above-described soxb1c-expressing ESNP population [51, 54]. Although amphioxus lacks definite placodes and neural crest, it has been proposed that these structures might have evolved via recruitment of the ventral ESN precursor field into the neural plate border $[51,93]$. Recent evidence from ascidian tunicates supports this notion: as in vertebrates, the PNS of the ascidian trunk and dorsal tail is derived from protoplacodal ectoderm adjacent to the neural plate that requires BMP inhibition for its specification, whereas peripheral ESNs of the ventral tail region are derived from a ventral ectoderm that requires high levels of BMP signaling, as in amphioxus [94-96]. Furthermore, caudal neural plate border cells of Ciona intestinalis were shown to include a migratory cell type resembling the neural crest-derived spinal ganglia neurons of vertebrates [97].

Of note, in early amphioxus larvae between 30 and $36 \mathrm{hpf}$, all ESNs showed GLU-ir, whereas later larvae also possessed GLU-negative ESNs. The GLU-ir ESNs of late larvae, between 48 and 108 hpf, were mostly concentrated in the mid-trunk ectoderm, i.e. in the area of the putative neurogenic niche. From these results, we hypothesize that most ESNs are born glutamatergic, but may later switch to other neurotransmitter identities. This hypothesis is supported by the downregulation of $t / x$ expression in some but not all differentiated ESNs of amphioxus larvae at $36 \mathrm{hpf}$. In vertebrates, TLX determines GLU phenotypes by suppressing GABA phenotypes [98]. GABA-ir ESNs have previously been described in amphioxus larvae between 36 and $108 \mathrm{hpf}[32,50]$. Accordingly, GABAergic fates are probably assigned to at least some amphioxus ESNs in a similar manner as in vertebrates. 
The generation of primarily GLU-ir sensory neurons from a neurogenic niche in the amphioxus ectoderm is comparable to the olfactory placode of vertebrates, which gives rise to olfactory receptor neurons that convey sensory information directly to the CNS via conventional glutamatergic synapses $[99,100]$. The olfactory placode comprises mostly slowly dividing neural progenitors [100]. Consistently, our EdU labeling results reveal only low levels of cell proliferation in the mid-trunk ectoderm of amphioxus larvae. In addition, it has been demonstrated that RA signaling is required for maintaining the slowly dividing neural precursor cells of the vertebrate olfactory epithelium [11]. This resembles RA signaling functions in regulating ESN production from the neurogenic niche in the amphioxus ectoderm. Although RA signaling has been implicated in patterning derivatives of other vertebrate placodes [17], it is currently unclear whether it also controls maintenance of their progenitor cells. Nonetheless, our findings support an evolutionary relationship between the soxb1c-expressing ESNPs in the lateral ectoderm of amphioxus larvae and vertebrate placodes.

Intriguingly, a role for RA signaling in maintaining slowly dividing neural precursor cells has further been confirmed in the developing and adult forebrain of mice [101]. Moreover, blocking of RA signaling was shown to reduce neural progenitor proliferation in the telencephalon of mice [102] as well as in the neural tube of quail and zebrafish embryos [103-105], whereas RA treatment increases the number of proliferating cells in chicken ventral neural tube explants [106]. Accordingly, RA signals are not only able to drive neural cell differentiation throughout much of the vertebrate nervous system [81, 82], but may exert either attenuating or stimulating effects that tune cell cycling behavior in a highly contextdependent manner to ensure a continuing production of neural cells without depleting the neural progenitor pool. The data presented in this study indicate that such a function exists also in amphioxus and is thus a conserved feature of chordate neurogenesis. The roles RA signaling plays in specifying and patterning the preplacodal region $[107,108]$ and in regulating the morphogenesis of specific placode derivatives, such as the olfactory [10, 11], adenohypophyseal [109], and otic placodes [12-14], hence appear to already be pre-figured in the amphioxus ectoderm. 


\section{Conclusions}

This study provides evidence for the presence of a neurogenic niche in the ectoderm of amphioxus larvae, which consists of soxb1c-expressing neural progenitors and ensures a continuous supply of ESNs during body elongation. Our data further support an important role for RA signaling in regulating the activity and the locally restricted maintenance of this neurogenic niche. Similar RA signaling functions can be observed in vertebrates [17], suggesting their conservation within chordates and indicating a close evolutionary relationship between the heredescribed neurogenic niche in the amphioxus ectoderm and vertebrate neurogenic placodes. Taken together, our findings thus illustrate the complex context-dependent actions of RA signaling during chordate PNS formation and provide novel insights into the evolution of this developmental process. 


\section{Declarations}

\section{Acknowledgements}

The authors would like to thank Thurston Lacalli, Nicholas D. Holland, and Linda Z. Holland for fruitful discussions. We are also grateful to Ram Reshef for his vital support with administrative issues.

\section{Funding}

This work was supported by a grant from the Agence Nationale de la Recherche (ANR-11-JSV2-002-01) and by funds from the Réseau André Picard (ANR-11-IDEX-0004-02, Sorbonne Universities) to MS and by a National Grant of the University of Genoa, Italy (2015) to SC. EZ was a doctoral fellow of the Studienstiftung der Deutschen Wirtschaft (SDW).

\section{Ethics approval and consent to participate \\ Not applicable.}

\section{Consent for publication}

Not applicable.

\section{Availability of data and material}

All data used in this study are included in this published article and its supplementary materials.

\section{Competing interests}

The authors declare that they have no competing interests.

\section{Authors' contributions}

EZ designed and performed experiments, analyzed and interpreted data, and wrote the manuscript. GG supported the collection of gene expression data, NSMR carried out phylogenetic analyses, and JKY contributed vital advice concerning the selection of candidate genes. JKY, JCC, and SC provided methodological assistance, supported data analyses, and commented the manuscript. MS designed and supervised the study, analyzed and interpreted data, and wrote the manuscript. All authors have read and approved the manuscript. 


\section{References}

1. Wang Y, Chen J, Du C, et al (2014) Characterization of retinoic acid-induced neurobehavioral effects in developing zebrafish. Environ Toxicol Chem 33:431-437 . doi: 10.1002/etc. 2453

2. Bailey JM, Oliveri AN, Karbhari N, et al (2016) Persistent behavioral effects following early life exposure to retinoic acid or valproic acid in zebrafish. NeuroToxicology 52:23-33 . doi: 10.1016/j.neuro.2015.10.001

3. Carta M, Stancampiano R, Tronci E, et al (2006) Vitamin A deficiency induces motor impairments and striatal cholinergic dysfunction in rats. Neuroscience 139:1163-1172 . doi: 10.1016/j.neuroscience.2006.01.027

4. Romand R, Krezel W, Beraneck M, et al (2013) Retinoic acid deficiency impairs the vestibular function. J Neurosci 33:5856-5866 . doi: 10.1523/JNEUROSCI.4618-12.2013

5. Srour M, Caron V, Pearson T, et al (2016) Gain-of-function mutations in RARB cause intellectual disability with progressive motor impairment: HUMAN MUTATION. Hum Mutat 37:786-793 . doi: 10.1002/humu.23004

6. Matt N, Dupé V, Garnier J-M, et al (2005) Retinoic acid-dependent eye morphogenesis is orchestrated by neural crest cells. Dev Camb Engl 132:4789-4800 . doi: 10.1242/dev.02031

7. Matt N, Ghyselinck NB, Pellerin I, Dupé V (2008) Impairing retinoic acid signalling in the neural crest cells is sufficient to alter entire eye morphogenesis. Dev Biol 320:140-148 . doi: 10.1016/j.ydbio.2008.04.039

8. Kumar S, Duester G (2010) Retinoic acid signaling in perioptic mesenchyme represses Wnt signaling via induction of Pitx2 and Dkk2. Dev Biol 340:67-74 . doi: 10.1016/j.ydbio.2010.01.027

9. Bohnsack BL, Kasprick DS, Kish PE, et al (2012) A zebrafish model of AxenfeldRieger syndrome reveals that pitx2 regulation by retinoic acid is essential for ocular and craniofacial development. Invest Ophthalmol Vis Sci 53:7-22 . doi: 10.1167/iovs.11-8494 10. Bhasin N, Maynard TM, Gallagher PA, LaMantia A-S (2003) Mesenchymal/epithelial regulation of retinoic acid signaling in the olfactory placode. Dev Biol 261:82-98

11. Paschaki M, Cammas L, Muta Y, et al (2013) Retinoic acid regulates olfactory progenitor cell fate and differentiation. Neural Develop 8:13 . doi: 10.1186/1749-8104-8-13

12. Romand R, Dollé P, Hashino E (2006) Retinoid signaling in inner ear development. J Neurobiol 66:687-704 . doi: 10.1002/neu.20244

13. Hans S, Christison J, Liu D, Westerfield M (2007) Fgf-dependent otic induction requires competence provided by Foxi1 and Dlx3b. BMC Dev Biol 7:5 . doi: 10.1186/1471213X-7-5

14. Thiede BR, Mann ZF, Chang W, et al (2014) Retinoic acid signalling regulates the development of tonotopically patterned hair cells in the chicken cochlea. Nat Commun 5:3840 . doi: $10.1038 /$ ncomms 4840

15. Simkin JE, Zhang D, Rollo BN, Newgreen DF (2013) Retinoic acid upregulates Ret and induces chain migration and population expansion in vagal neural crest cells to colonise the embryonic gut. PLoS ONE 8:e64077 . doi: 10.1371/journal.pone.0064077

16. Laudet V, Zieger E, Schubert M (2015) Evolution of the retinoic acid signaling pathway. In: Dollé P, Neiderreither K (eds) The retinoids. John Wiley \& Sons, Inc, Hoboken, NJ, pp 75-90

17. Zieger E, Schubert M (2017) New insights into the roles of retinoic acid signaling in nervous system development and the establishment of neurotransmitter systems. In:

International Review of Cell and Molecular Biology. Elsevier, pp 1-84

18. Chambon P (1996) A decade of molecular biology of retinoic acid receptors. FASEB J 10:940-954 
19. Moutier E, Ye T, Choukrallah M-A, et al (2012) Retinoic acid receptors recognize the mouse genome through binding elements with diverse spacing and topology. J Biol Chem 287:26328-26341 . doi: 10.1074/jbc.M112.361790

20. Vilhais-Neto GC, Pourquié O (2008) Retinoic acid. Curr Biol 18:R191-R192 . doi: 10.1016/j.cub.2007.12.042

21. Gronemeyer H, Gustafsson J-A, Laudet V (2004) Principles for modulation of the nuclear receptor superfamily. Nat Rev Drug Discov 3:950-964 . doi: 10.1038/nrd1551

22. Rhinn M, Dollé P (2012) Retinoic acid signalling during development. Development 139:843-858 . doi: 10.1242/dev.065938

23. Pennati R, Dell'Anna A, Zega G, et al (2013) Retinoic acid influences antero-posterior positioning of peptidergic neurons in the planula larva of the hydrozoan Clava multicornis. Mar Ecol 34:143-152 . doi: 10.1111/maec.12032

24. Farrar NR, Dmetrichuk JM, Carlone RL, Spencer GE (2009) A novel, nongenomic mechanism underlies retinoic acid-induced growth cone turning. J Neurosci 29:14136-14142 . doi: 10.1523/JNEUROSCI.2921-09.2009

25. Carter CJ, Farrar N, Carlone RL, Spencer GE (2010) Developmental expression of a molluscan RXR and evidence for its novel, nongenomic role in growth cone guidance. Dev Biol 343:124-137 . doi: 10.1016/j.ydbio.2010.03.023

26. Carter CJ, Rand C, Mohammad I, et al (2015) Expression of a retinoic acid receptor (RAR)-like protein in the embryonic and adult nervous system of a protostome species. J Exp Zoolog B Mol Dev Evol 324:51-67 . doi: 10.1002/jez.b.22604

27. Sasakura Y, Kanda M, Ikeda T, et al (2012) Retinoic acid-driven Hoxl is required in the epidermis for forming the otic/atrial placodes during ascidian metamorphosis.

Development 139:2156-2160 . doi: 10.1242/dev.080234

28. Pasini A, Manenti R, Rothbächer U, Lemaire P (2012) Antagonizing retinoic acid and FGF/MAPK pathways control posterior body patterning in the invertebrate chordate Ciona intestinalis. PLoS ONE 7:e46193 . doi: 10.1371/journal.pone.0046193

29. Schubert M, Holland ND, Escriva H, et al (2004) Retinoic acid influences anteroposterior positioning of epidermal sensory neurons and their gene expression in a developing chordate (amphioxus). Proc Natl Acad Sci 101:10320-10325 . doi:

10.1073/pnas.0403216101

30. Schubert M, Escriva H, Xavier-Neto J, Laudet V (2006) Amphioxus and tunicates as evolutionary model systems. Trends Ecol Evol 21:269-277 . doi: 10.1016/j.tree.2006.01.009 31. Onai T, Lin H-C, Schubert $M$, et al (2009) Retinoic acid and Wnt/ $\beta$-catenin have complementary roles in anterior/posterior patterning embryos of the basal chordate amphioxus. Dev Biol 332:223-233 . doi: 10.1016/j.ydbio.2009.05.571

32. Zieger E, Candiani S, Garbarino G, et al (2017) Roles of retinoic acid signaling in shaping the neuronal architecture of the developing amphioxus nervous system. Mol Neurobiol. doi: 10.1007/s12035-017-0727-8

33. Delsuc F, Brinkmann H, Chourrout D, Philippe H (2006) Tunicates and not cephalochordates are the closest living relatives of vertebrates. Nature 439:965-968 . doi: 10.1038/nature04336

34. Bertrand S, Escriva H (2011) Evolutionary crossroads in developmental biology: amphioxus. Development 138:4819-4830 . doi: 10.1242/dev.066720

35. Yue J-X, Yu J-K, Putnam NH, Holland LZ (2014) The transcriptome of an amphioxus, Asymmetron lucayanum, from the Bahamas: a window into chordate evolution. Genome Biol Evol 6:2681-2696 . doi: 10.1093/gbe/evu212

36. Holland LZ (2015) Evolution of basal deuterostome nervous systems. J Exp Biol 218:637-645 . doi: 10.1242/jeb.109108

37. Bourlat SJ, Juliusdottir T, Lowe CJ, et al (2006) Deuterostome phylogeny reveals 
monophyletic chordates and the new phylum Xenoturbellida. Nature 444:85-88 . doi: 10.1038/nature05241

38. Putnam NH, Butts T, Ferrier DEK, et al (2008) The amphioxus genome and the evolution of the chordate karyotype. Nature 453:1064-1071 . doi: 10.1038/nature06967 39. Holland LZ (2013) Evolution of new characters after whole genome duplications: insights from amphioxus. Semin Cell Dev Biol 24:101-109 . doi:

10.1016/j.semcdb.2012.12.007

40. Holland LZ (2015) Genomics, evolution and development of amphioxus and tunicates: the Goldilocks principle. J Exp Zoolog B Mol Dev Evol 324:342-352 . doi: 10.1002/jez.b.22569

41. Escriva H, Holland ND, Gronemeyer H, et al (2002) The retinoic acid signaling pathway regulates anterior/posterior patterning in the nerve cord and pharynx of amphioxus, a chordate lacking neural crest. Dev Camb Engl 129:2905-2916

42. Schubert M, Holland ND, Laudet V, Holland LZ (2006) A retinoic acid-Hox hierarchy controls both anterior/posterior patterning and neuronal specification in the developing central nervous system of the cephalochordate amphioxus. Dev Biol 296:190-202 . doi:

10.1016/j.ydbio.2006.04.457

43. Koop D, Holland ND, Sémon M, et al (2010) Retinoic acid signaling targets Hox genes during the amphioxus gastrula stage: insights into early anterior-posterior patterning of the chordate body plan. Dev Biol 338:98-106 . doi: 10.1016/j.ydbio.2009.11.016

44. Albuixech-Crespo B, López-Blanch L, Burguera D, et al (2017) Molecular regionalization of the developing amphioxus neural tube challenges major partitions of the vertebrate brain. PLOS Biol 15:e2001573 . doi: 10.1371/journal.pbio.2001573

45. Wicht H, Lacalli TC (2005) The nervous system of amphioxus: structure, development, and evolutionary significance. Can J Zool 83:122-150 . doi: 10.1139/z04-163 46. Zieger E, Lacalli TC, Pestarino M, et al (in press) The origin of dopaminergic systems in chordate brains: insights from amphioxus. Int J Dev Biol

47. Holland LZ (2015) The origin and evolution of chordate nervous systems. Philos Trans R Soc B Biol Sci 370:20150048-20150048 . doi: 10.1098/rstb.2015.0048

48. Le Petillon Y, Luxardi G, Scerbo P, et al (2017) Nodal-Activin pathway is a conserved neural induction signal in chordates. Nat Ecol Evol 1:1192-1200 . doi: $10.1038 / \mathrm{s} 41559-017-0226-3$

49. Kaltenbach SL, Yu J-K, Holland ND (2009) The origin and migration of the earliestdeveloping sensory neurons in the peripheral nervous system of amphioxus. Evol Dev 11:142-151 . doi: 10.1111/j.1525-142X.2009.00315.x

50. Lu T-M, Luo Y-J, Yu J-K (2012) BMP and Delta/Notch signaling control the development of amphioxus epidermal sensory neurons: insights into the evolution of the peripheral sensory system. Development 139:2020-2030 . doi: 10.1242/dev.073833

51. Meulemans D, Bronner-Fraser M (2007) The amphioxus SoxB family: implications for the evolution of vertebrate placodes. Int J Biol Sci 3:356-364

52. Satoh G, Wang Y, Zhang P, Satoh N (2001) Early development of amphioxus nervous system with special reference to segmental cell organization and putative sensory cell precursors: a study based on the expression of pan-neuronal marker gene Hu/elav. J Exp Zool 291:354-364

53. Mazet F, Masood S, Luke GN, et al (2004) Expression of AmphiCoe, an amphioxus $\mathrm{COE} / \mathrm{EBF}$ gene, in the developing central nervous system and epidermal sensory neurons. genesis 38:58-65 . doi: 10.1002/gene.20006

54. Kozmik Z, Holland ND, Kreslova J, et al (2007) Pax-Six-Eya-Dach network during amphioxus development: conservation in vitro but context specificity in vivo. Dev Biol 306:143-159 . doi: 10.1016/j.ydbio.2007.03.009 
55. Holland ND, Yu J-K (2002) Epidermal receptor development and sensory pathways in vitally stained amphioxus (Branchiostoma floridae): amphioxus sensory neurone development. Acta Zool 83:309-319 . doi: 10.1046/j.1463-6395.2002.00120.x 56. Candiani S, Moronti L, Ramoino P, et al (2012) A neurochemical map of the developing amphioxus nervous system. BMC Neurosci 13:59 . doi: 10.1186/1471-2202-13-59 57. Fuentes M, Schubert M, Dalfo D, et al (2004) Preliminary observations on the spawning conditions of the European amphioxus (Branchiostoma lanceolatum) in captivity. $\mathrm{J}$ Exp Zoolog B Mol Dev Evol 302:384-391 . doi: 10.1002/jez.b.20025

58. Fuentes M, Benito E, Bertrand S, et al (2007) Insights into spawning behavior and development of the European amphioxus Branchiostoma lanceolatum. J Exp Zoolog B Mol Dev Evol 308:484-493 . doi: 10.1002/jez.b.21179

59. Theodosiou M, Colin A, Schulz J, et al (2011) Amphioxus spawning behavior in an artificial seawater facility. J Exp Zoolog B Mol Dev Evol 316:263-275 . doi:

10.1002/jez.b.21397

60. Holland LZ, Yu J-K (2004) Cephalochordate (amphioxus) embryos: procurement, culture, and basic methods. Methods Cell Biol 74:195-215

61. Schneider CA, Rasband WS, Eliceiri KW (2012) NIH Image to ImageJ: 25 years of image analysis. Nat Methods 9:671-675

62. Oulion S, Bertrand S, Belgacem MR, et al (2012) Sequencing and analysis of the Mediterranean amphioxus (Branchiostoma lanceolatum) transcriptome. PloS One 7:e36554 . doi: 10.1371/journal.pone.0036554

63. Edgar RC (2004) MUSCLE: multiple sequence alignment with high accuracy and high throughput. Nucleic Acids Res 32:1792-1797 . doi: 10.1093/nar/gkh340

64. Capella-Gutierrez S, Silla-Martinez JM, Gabaldon T (2009) trimAl: a tool for automated alignment trimming in large-scale phylogenetic analyses. Bioinformatics 25:19721973 . doi: 10.1093/bioinformatics/btp348

65. Stamatakis A, Hoover P, Rougemont J (2008) A rapid bootstrap algorithm for the RAxML web servers. Syst Biol 57:758-771 . doi: 10.1080/10635150802429642

66. Darriba D, Taboada GL, Doallo R, Posada D (2011) ProtTest 3: fast selection of bestfit models of protein evolution. Bioinformatics 27:1164-1165 . doi:

10.1093/bioinformatics/btr088

67. Yu JKS, Holland LZ (2009) Extraction of RNA from amphioxus embryos or adult amphioxus tissue. Cold Spring Harb Protoc 2009:pdb.prot5288 . doi: 10.1101/pdb.prot5288 68. Yu JKS, Holland LZ (2009) Amphioxus whole-mount in situ hybridization. Cold Spring Harb Protoc 2009:pdb.prot5286 . doi: 10.1101/pdb.prot5286

69. Bayascas JR, Yuste VJ, Benito E, et al (2002) Isolation of AmphiCASP-3/7, an ancestral caspase from amphioxus (Branchiostoma floridae). Evolutionary considerations for vertebrate caspases. Cell Death Differ 9:1078-1089 . doi: 10.1038/sj.cdd.4401075

70. Stokes MD (1997) Larval locomotion of the lancelet Branchiostoma floridae. J Exp Biol 200:1661-1680

71. McEntee WJ, Crook TH (1993) Glutamate: its role in learning, memory, and the aging brain. Psychopharmacology (Berl) 111:391-401

72. Benito-Gutiérrez E (2006) A gene catalogue of the amphioxus nervous system. Int J Biol Sci 149-160 . doi: 10.7150/ijbs.2.149

73. Lacalli TC (2004) Sensory systems in amphioxus: a window on the ancestral chordate condition. Brain Behav Evol 64:148-162 . doi: 10.1159/000079744

74. Lacalli TC, Gilmour THJ, Kelly SJ (1999) The oral nerve plexus in amphioxus larvae: function, cell types and phylogenetic significance. Proc R Soc B Biol Sci 266:1461-1470 . doi: 10.1098/rspb.1999.0801

75. Benito-Gutiérrez È, Illas M, Comella JX, Garcia-Fernàndez J (2005) Outlining the 
nascent nervous system of Branchiostoma floridae (amphioxus) by the pan-neural marker AmphiElav. Brain Res Bull 66:518-521 . doi: 10.1016/j.brainresbull.2005.03.007

76. Sobreira TJP, Marletaz F, Simoes-Costa M, et al (2011) Structural shifts of aldehyde dehydrogenase enzymes were instrumental for the early evolution of retinoid-dependent axial patterning in metazoans. Proc Natl Acad Sci 108:226-231 . doi: 10.1073/pnas.1011223108 77. Carvalho JE, Theodosiou M, Chen J, et al (2017) Lineage-specific duplication of amphioxus retinoic acid degrading enzymes (CYP26) resulted in sub-functionalization of patterning and homeostatic roles. BMC Evol Biol 17: . doi: 10.1186/s12862-016-0863-1 78. Shimozono S, Iimura T, Kitaguchi T, et al (2013) Visualization of an endogenous retinoic acid gradient across embryonic development. Nature 496:363-366 . doi:

10.1038 /nature12037

79. Scott SH (2004) Optimal feedback control and the neural basis of volitional motor control. Nat Rev Neurosci 5:532-546 . doi: 10.1038/nrn1427

80. Windhorst U (2007) Muscle proprioceptive feedback and spinal networks. Brain Res Bull 73:155-202 . doi: 10.1016/j.brainresbull.2007.03.010

81. Duester G (2013) Retinoid signaling in control of progenitor cell differentiation during mouse development. Semin Cell Dev Biol 24:694-700 . doi: 10.1016/j.semcdb.2013.08.001

82. Janesick A, Wu SC, Blumberg B (2015) Retinoic acid signaling and neuronal differentiation. Cell Mol Life Sci. doi: 10.1007/s00018-014-1815-9

83. Gudas LJ (2013) Retinoids induce stem cell differentiation via epigenetic changes. Semin Cell Dev Biol 24:701-705 . doi: 10.1016/j.semcdb.2013.08.002

84. Schug TT, Berry DC, Shaw NS, et al (2007) Dual transcriptional activities underlie opposing effects of retinoic acid on cell survival. Cell 129:723

85. Wolf G (2008) Retinoic acid as cause of cell proliferation or cell growth inhibition depending on activation of one of two different nuclear receptors. Nutr Rev 66:55-59 . doi: 10.1111/j.1753-4887.2007.00006.x

86. Richards GS, Rentzsch F (2014) Transgenic analysis of a SoxB gene reveals neural progenitor cells in the cnidarian Nematostella vectensis. Development 141:4681-4689 . doi: 10.1242/dev.112029

87. Stollewerk A (2016) A flexible genetic toolkit for arthropod neurogenesis. Philos Trans R Soc B Biol Sci 371:20150044 . doi: 10.1098/rstb.2015.0044

88. Burke RD, Moller DJ, Krupke OA, Taylor VJ (2014) Sea urchin neural development and the metazoan paradigm of neurogenesis: Embryonic Neurogenesis. genesis 52:208-221 . doi: $10.1002 /$ dvg. 22750

89. Garner S, Zysk I, Byrne G, et al (2016) Neurogenesis in sea urchin embryos and the diversity of deuterostome neurogenic mechanisms. Development 143:286-297 . doi:

$10.1242 /$ dev. 124503

90. Cunningham D, Casey ES (2014) Spatiotemporal development of the embryonic nervous system of Saccoglossus kowalevskii. Dev Biol 386:252-263 . doi:

10.1016/j.ydbio.2013.12.001

91. Hartenstein V, Stollewerk A (2015) The evolution of early neurogenesis. Dev Cell 32:390-407 . doi: 10.1016/j.devcel.2015.02.004

92. Koop D, Chen J, Theodosiou M, et al (2014) Roles of retinoic acid and Tbx1/10 in pharyngeal segmentation: amphioxus and the ancestral chordate condition. EvoDevo 5:36 . doi: 10.1186/2041-9139-5-36

93. Holland LZ (2009) Chordate roots of the vertebrate nervous system: expanding the molecular toolkit. Nat Rev Neurosci 10:736-746 . doi: 10.1038/nrn2703

94. Ohtsuka Y, Matsumoto J, Katsuyama Y, Okamura Y (2014) Nodal signaling regulates specification of ascidian peripheral neurons through control of the BMP signal. Development 141:3889-3899 . doi: 10.1242/dev.110213 
95. Waki K, Imai KS, Satou Y (2015) Genetic pathways for differentiation of the peripheral nervous system in ascidians. Nat Commun 6:8719 . doi: 10.1038/ncomms9719 96. Abitua PB, Gainous TB, Kaczmarczyk AN, et al (2015) The pre-vertebrate origins of neurogenic placodes. Nature 524:462-465 . doi: 10.1038/nature14657

97. Stolfi A, Ryan K, Meinertzhagen IA, Christiaen L (2015) Migratory neuronal progenitors arise from the neural plate borders in tunicates. Nature 527:371-374 . doi: 10.1038 /nature 15758

98. Cheng L, Arata A, Mizuguchi R, et al (2004) Tlx3 and Tlx1 are post-mitotic selector genes determining glutamatergic over GABAergic cell fates. Nat Neurosci 7:510-517 . doi: $10.1038 / \mathrm{nn} 1221$

99. Murphy GJ, Glickfeld LL, Balsen Z, Isaacson JS (2004) Sensory neuron signaling to the brain: properties of transmitter release from olfactory nerve terminals. J Neurosci 24:3023-3030 . doi: 10.1523/JNEUROSCI.5745-03.2004

100. Tucker ES, Lehtinen MK, Maynard T, et al (2010) Proliferative and transcriptional identity of distinct classes of neural precursors in the mammalian olfactory epithelium. Development 137:2471-2481 . doi: 10.1242/dev.049718

101. Haskell GT, LaMantia A-S (2005) Retinoic acid signaling identifies a distinct precursor population in the developing and adult forebrain. J Neurosci 25:7636-7647 . doi: 10.1523/JNEUROSCI.0485-05.2005

102. Rajaii F, Bitzer ZT, Xu Q, Sockanathan S (2008) Expression of the dominant negative retinoid receptor, RAR403, alters telencephalic progenitor proliferation, survival, and cell fate specification. Dev Biol 316:371-382 . doi: 10.1016/j.ydbio.2008.01.041

103. Wilson L, Gale E, Maden M (2003) The role of retinoic acid in the morphogenesis of the neural tube. J Anat 203:357-368 . doi: 10.1046/j.1469-7580.2003.00230.x 104. England S, Batista MF, Mich JK, et al (2011) Roles of Hedgehog pathway components and retinoic acid signalling in specifying zebrafish ventral spinal cord neurons. Development 138:5121-5134 . doi: 10.1242/dev.066159

105. Mich JK, Chen JK (2011) Hedgehog and retinoic acid signaling cooperate to promote motoneurogenesis in zebrafish. Development 138:5113-5119 . doi: 10.1242/dev.066225

106. Sockanathan S, Jessell TM (1998) Motor neuron-derived retinoid signaling specifies the subtype identity of spinal motor neurons. Cell 94:503-514 . doi: 10.1016/S0092-

8674(00)81591-3

107. Janesick A, Shiotsugu J, Taketani M, Blumberg B (2012) RIPPLY3 is a retinoic acidinducible repressor required for setting the borders of the pre-placodal ectoderm.

Development 139:1213-1224 . doi: 10.1242/dev.071456

108. Jaurena MB, Juraver-Geslin H, Devotta A, Saint-Jeannet J-P (2015) Zic 1 controls placode progenitor formation non-cell autonomously by regulating retinoic acid production and transport. Nat Commun 6:7476 . doi: 10.1038/ncomms8476

109. Maden M, Blentic A, Reijntjes S, et al (2007) Retinoic acid is required for specification of the ventral eye field and for Rathke's pouch in the avian embryo. Int J Dev Biol 51:191-200 . doi: 10.1387/ijdb.062175mm 


\section{Supplementary material}

Additional file 1: Movie showing reactions of amphioxus larvae at $48 \mathrm{hpf}$ (hours post fertilization) to mechanical stimulation. Response $A=$ quick muscular swimming movement away from the stimulus. Response $B=$ intense wiggling and bending movements without clear directionality. Response $C=$ short wiggling motion on the spot. Response $D=$ short disconnected twitches or bends on the spot. Response $E=$ no visible reaction.

Additional file 2: Movie showing responses of amphioxus larvae at $48 \mathrm{hpf}$ (hours post fertilization) to chemical stimulation. As indicated in the movie, the amphioxus embryos were exposed to DMSO (Control), the retinoic acid receptor (RAR) antagonist BMS493 or all-trans retinoic acid (RA), starting from treatment time points (t) at 6 or $24 \mathrm{hpf}$. Upon reaching the $48 \mathrm{hpf}$ stage, the larvae were further exposed to agarose blocks, which had either been dissolved in artificial seawater (negative control) or in artificial seawater supplemented with $0.1 \mathrm{M} \mathrm{L-glutamate.}$

Additional file 3: Figure S1. Effects of retinoic acid (RA) signaling on glutamate chemoreception and larval circling/spiraling behavior in amphioxus. Amphioxus embryos were exposed to DMSO (Control, green bars), the RA receptor (RAR) antagonist BMS493 (blue bars) or all-trans RA (red bars), starting from treatment time points (t) at 6 or $24 \mathrm{hpf}$ (hours post fertilization). Subsequently, by $48 \mathrm{hpf}$, the animals were further exposed to agarose blocks, which had either been dissolved in artificial seawater (negative control, dark-colored bars) or in artificial seawater supplemented with $0.1 \mathrm{M} \mathrm{L-glutamate} \mathrm{(light-colored} \mathrm{bars).} \mathrm{The} \mathrm{size} \mathrm{of} \mathrm{the} \mathrm{colored}$ bars indicates the average number of circles amphioxus larvae swam without interruption and the error bars indicate the standard deviation $(\sigma)$. The total number $(\mathrm{n})$ of animals counted is given at the base of each colored bar. Asterisks $\left({ }^{*}\right)$ above an error bar indicate that the difference between this condition and the corresponding control is statistically significant with a p-value $<0.05$ (one asterisk, ${ }^{*}$ ) or with a pvalue $<0.01$ (two asterisks, ${ }^{* *}$ ). Only larvae that had passed by the agarose block within a $1 \mathrm{~cm}$ radius and within 0.5 to $10 \mathrm{~min}$ after introduction of the agarose block 
were taken into consideration. For RA treatments at $6 \mathrm{hpf}$, circling/spiraling was rarely observed, precluding statistical analyses.

Additional file 4: Figure S2. Effects of retinoic acid (RA) signaling on the development of vglut-expressing cells in amphioxus. Larvae are shown in lateral (a-i) or dorsal (d'-i') view with their anterior ends directed toward the right. Developmental stages are given as hours post fertilization (hpf). Embryos have been treated from the treatment stage (t) at $6 \mathrm{hpf}$ with DMSO (Control), the RA receptor (RAR) antagonist BMS493 or all-trans RA, as indicated. All scale bars are $50 \mu \mathrm{m}$. The scale bar in (a) applies also to b,c, the scale bar in $\mathbf{d}$ applies also to d',e,e',f,f', and the scale bar in $\mathbf{g}$ applies also to $\mathbf{g}^{\prime}, \mathbf{h}, \mathbf{h}^{\prime}, \mathbf{i}, \mathbf{i}$ '.

Additional file 5: Figure S3. Expression of hu/elav during amphioxus development. a-e Lateral views of amphioxus embryos and larvae at different developmental stages from 15 to $36 \mathrm{hpf}$ (hours post fertilization). Anterior ends are directed toward the right. a'-e' Dorsal views of the amphioxus embryos and larvae shown respectively in a-e. c-e Dotted boxes indicate the ectodermal domain containing a conspicuously high density of hu/elav-expressing ectodermal sensory neuron progenitors (ESNPs). All scale bars are $50 \mu \mathrm{m}$. The scale bar in a, b, c, d, and e respectively also applies to a', b', c', d', and e'.

Additional file 6: Figure S4. Expression of $t / x$ during amphioxus development. a-e Lateral views of amphioxus embryos and larvae at different developmental stages from 15 to $36 \mathrm{hpf}$ (hours post fertilization). Anterior ends are directed towards the right. a'-e' Dorsal views of the amphioxus embryos and larvae shown respectively in a-e. c-e Dotted boxes indicate the ectodermal domain containing a conspicuously high density of ectodermal sensory neurons (ESNs) (compare with Figure 3), hu/elav-expressing ESN progenitors (ESNPs) (compare with Additional file 5: Figure S3) as well as soxb1c-expressing ESNPs (compare with Additional file 7: Figure S5), 
but not $t / x$-expressing ESNPs. All scale bars are $50 \mu \mathrm{m}$. The scale bar in a, b, c, d, and e respectively also applies to a', b', c', d', and e'.

Additional file 7: Figure S5. Expression of soxb1c during amphioxus development. a-e Lateral views of amphioxus embryos and larvae at different developmental stages from 15 to $36 \mathrm{hpf}$ (hours post fertilization). The anterior is directed towards the right. a'-e' Dorsal views of the amphioxus embryos and larvae shown respectively in a-e. The images in c-e' are focused on soxb1c expression in the ectoderm. Dotted boxes indicate the ectodermal domain containing a conspicuously high density of soxb1c-expressing ectodermal sensory neuron progenitors (ESNPs). All scale bars are $50 \mu \mathrm{m}$. The scale bar in $\mathbf{a}, \mathbf{b}, \mathbf{c}, \mathbf{d}$, and e respectively also applies to a', b', c', d', and $\mathbf{e}^{\prime}$.

Additional file 8: Figure S6. Maximum-likelihood phylogenies for a ELAV, b TLX and c SOXB1. Trees were inferred using RAxML with 1000 rapid bootstraps. 
Additional file 9: Table S1. GenBank accession numbers of sequences used for phylogenetic analyses.

\begin{tabular}{|c|c|c|}
\hline Species & Gene & $\begin{array}{l}\text { Accession } \\
\text { number }\end{array}$ \\
\hline \multirow[t]{3}{*}{ Homo sapiens } & $\begin{array}{l}\text { Elav1 } \\
\text { Elav2 } \\
\text { Elav3 } \\
\text { Elav4 }\end{array}$ & $\begin{array}{l}\text { NP_001410.2 } \\
\text { NP_004423.2 } \\
\text { NP_001411.2 } \\
\text { NP_068771.2 }\end{array}$ \\
\hline & $\begin{array}{l}T / x 1 \\
T I \times 2 \\
T I \times 3\end{array}$ & $\begin{array}{l}\text { NP_005512.1 } \\
\text { NP_057254.1 } \\
\text { NP_066305.2 }\end{array}$ \\
\hline & $\begin{array}{l}\text { Sox1 } \\
\text { Sox2 } \\
\text { Sox3 }\end{array}$ & $\begin{array}{l}\text { NP_005977.2 } \\
\text { NP_003097.1 } \\
\text { NP_005625.2 }\end{array}$ \\
\hline \multirow[t]{3}{*}{ Gallus gallus } & $\begin{array}{l}\text { Elav1 } \\
\text { Elav2 } \\
\text { Elav3 } \\
\text { Elav4 }\end{array}$ & $\begin{array}{l}\text { NP_990164.1 } \\
\text { XP_004949594.1 } \\
\text { NP_990163.1 } \\
\text { NP_990161.1 }\end{array}$ \\
\hline & $\begin{array}{l}T / \times 1 \\
T I \times 2 \\
T I \times 3\end{array}$ & $\begin{array}{l}\text { NP_990346.1 } \\
\text { XP_004949910.2 } \\
\text { XP_015149103.1 }\end{array}$ \\
\hline & $\begin{array}{l}\text { Sox1 } \\
\text { Sox2 } \\
\text { Sox3 }\end{array}$ & $\begin{array}{l}\text { NP_989664.1 } \\
\text { NP_990519.2 } \\
\text { NP_989526.1 }\end{array}$ \\
\hline \multirow{5}{*}{$\begin{array}{l}\text { Branchiostoma } \\
\text { floridae }\end{array}$} & Hu/elav & XP_002605964.1 \\
\hline & $T / x$ & XP_002609066.1 \\
\hline & Soxb1a & AF271787 \\
\hline & Soxb1b & XP_002592065.1 \\
\hline & Soxb1c & ABG66527.1 \\
\hline
\end{tabular}




\begin{tabular}{|l|l|l|}
\hline \multirow{3}{*}{$\begin{array}{l}\text { Branchiostoma } \\
\text { lanceolatum }\end{array}$} & Hu/elav & KY569299 \\
\cline { 2 - 3 } & Tlx & KY569298 \\
\cline { 2 - 3 } & Soxb1c & KY569300 \\
\hline Strongylocentrotus & Elav & XP_802063.2 \\
\cline { 2 - 3 } & TIx & XP_781741.3 \\
\cline { 2 - 3 } & Soxb1 & NP_999639.1 \\
\hline Tribolium castaneum & Elav & XP_008200562.1 \\
\cline { 2 - 3 } & Tlx & XP_008198314.1 \\
\cline { 2 - 3 } & SoxNeuro & XP_008194311.1 \\
\hline $\begin{array}{l}\text { Drosophila } \\
\text { melanogaster }\end{array}$ & Elav & NP_525033.1 \\
& Rbp9 & NP_599124.1 \\
& Fne & NP_572842.1 \\
\cline { 2 - 3 } & Tlx & NP_476873.2 \\
\cline { 2 - 3 } & SoxNeuro & Q9U1H5 \\
\hline
\end{tabular}


Control t6 BMS493

t6 RA

t24 BMS493 t24 RA
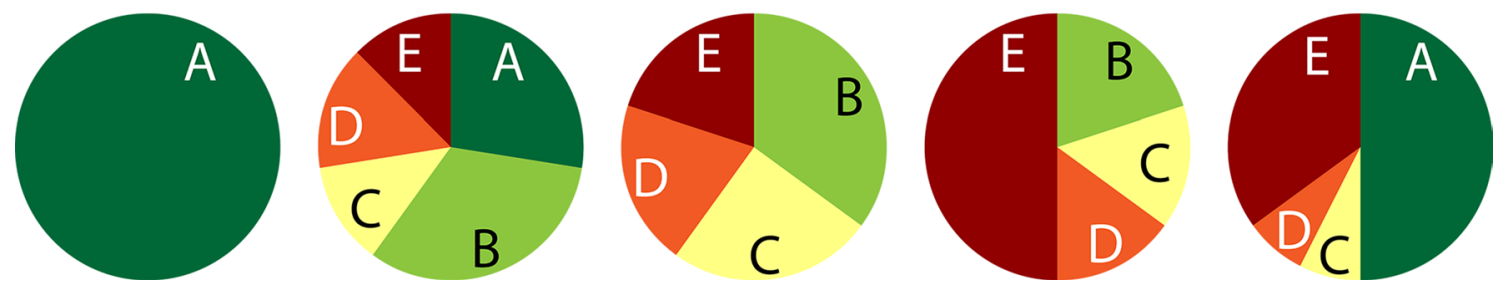
$\mathbf{a}$

$a$

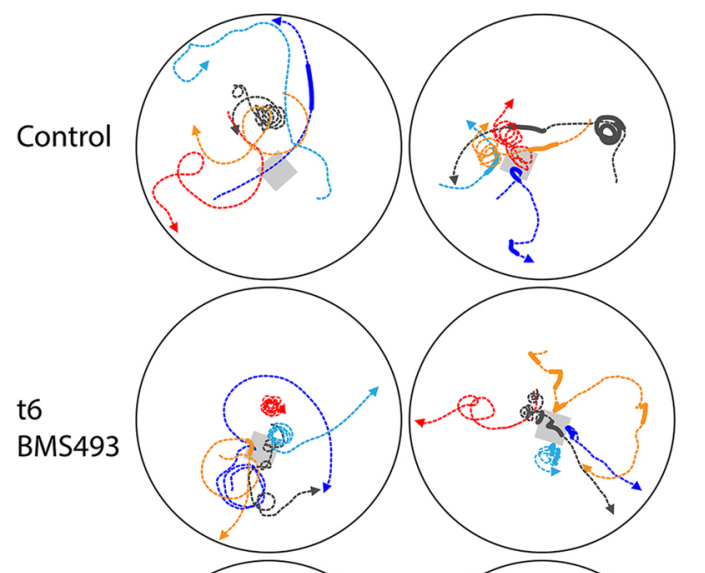

t24

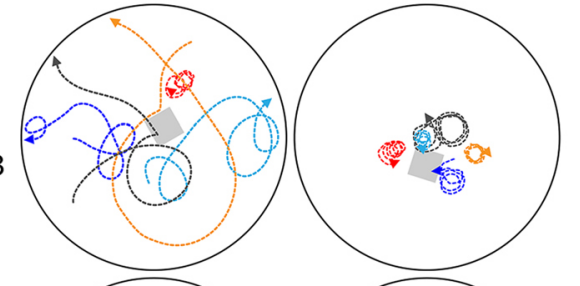

t6 RA

t24 RA

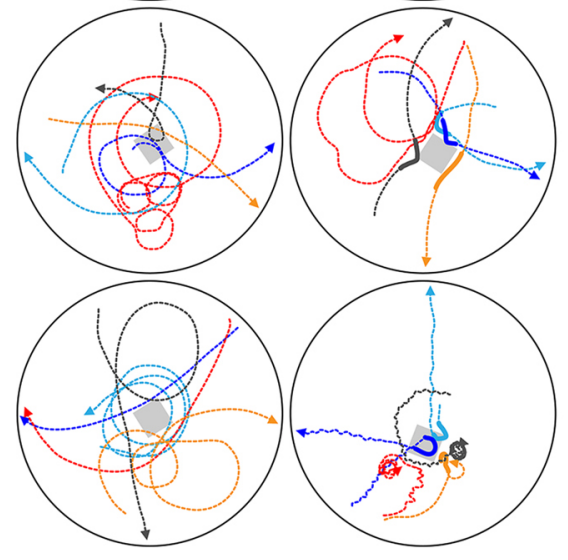

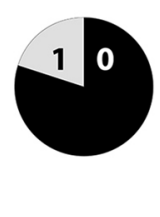
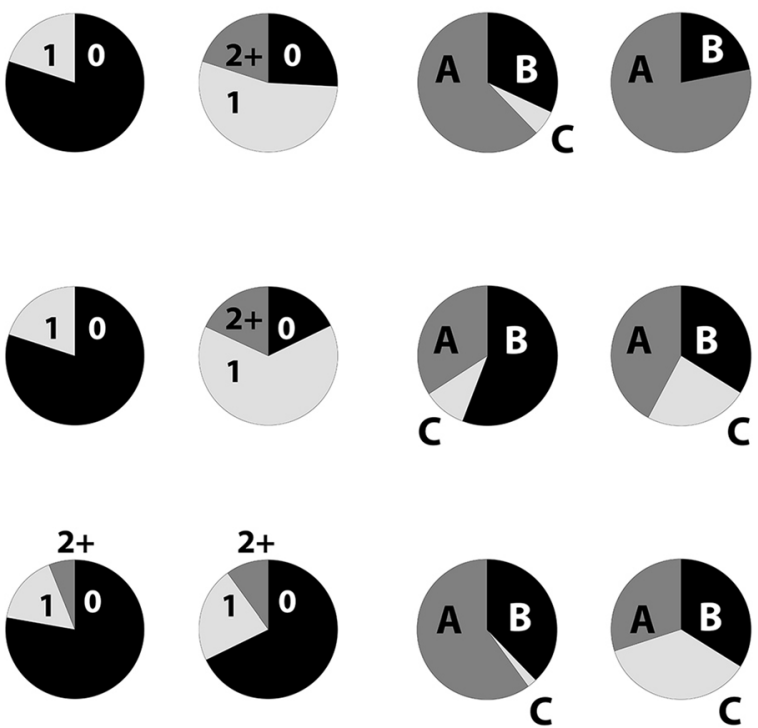

C
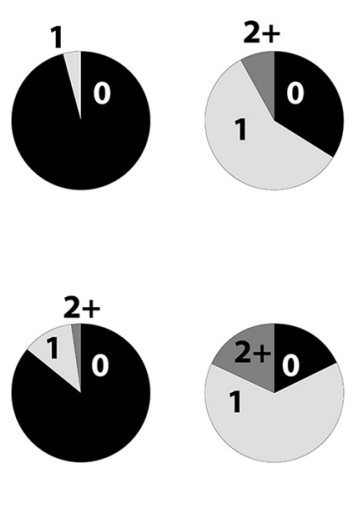
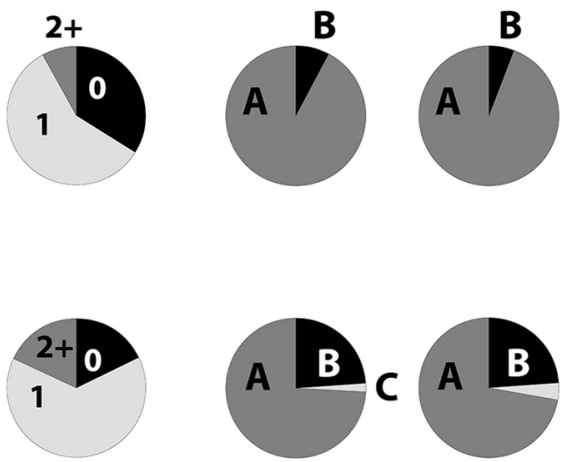

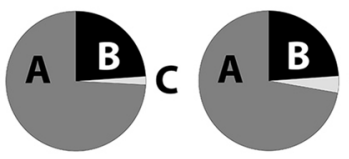




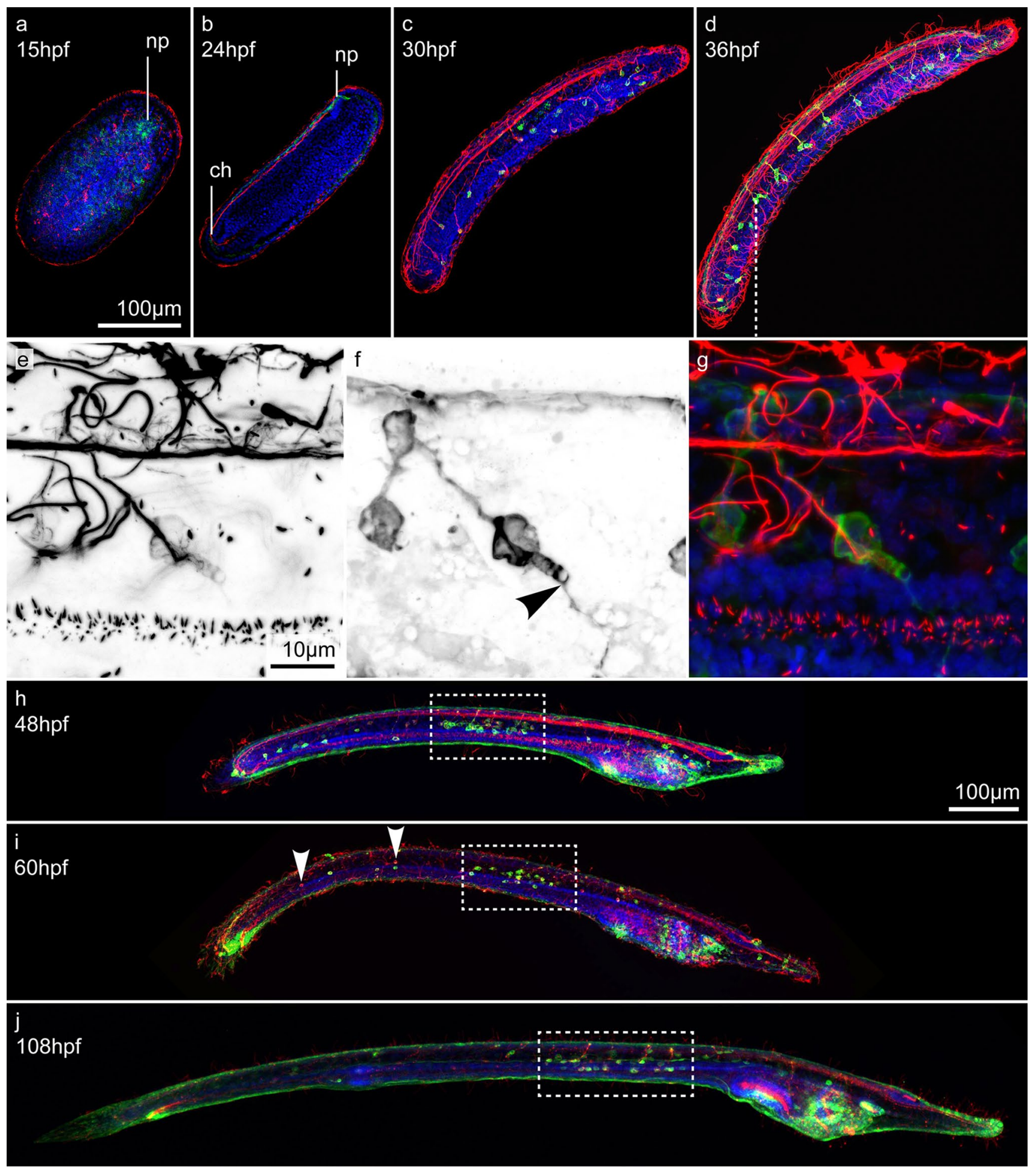




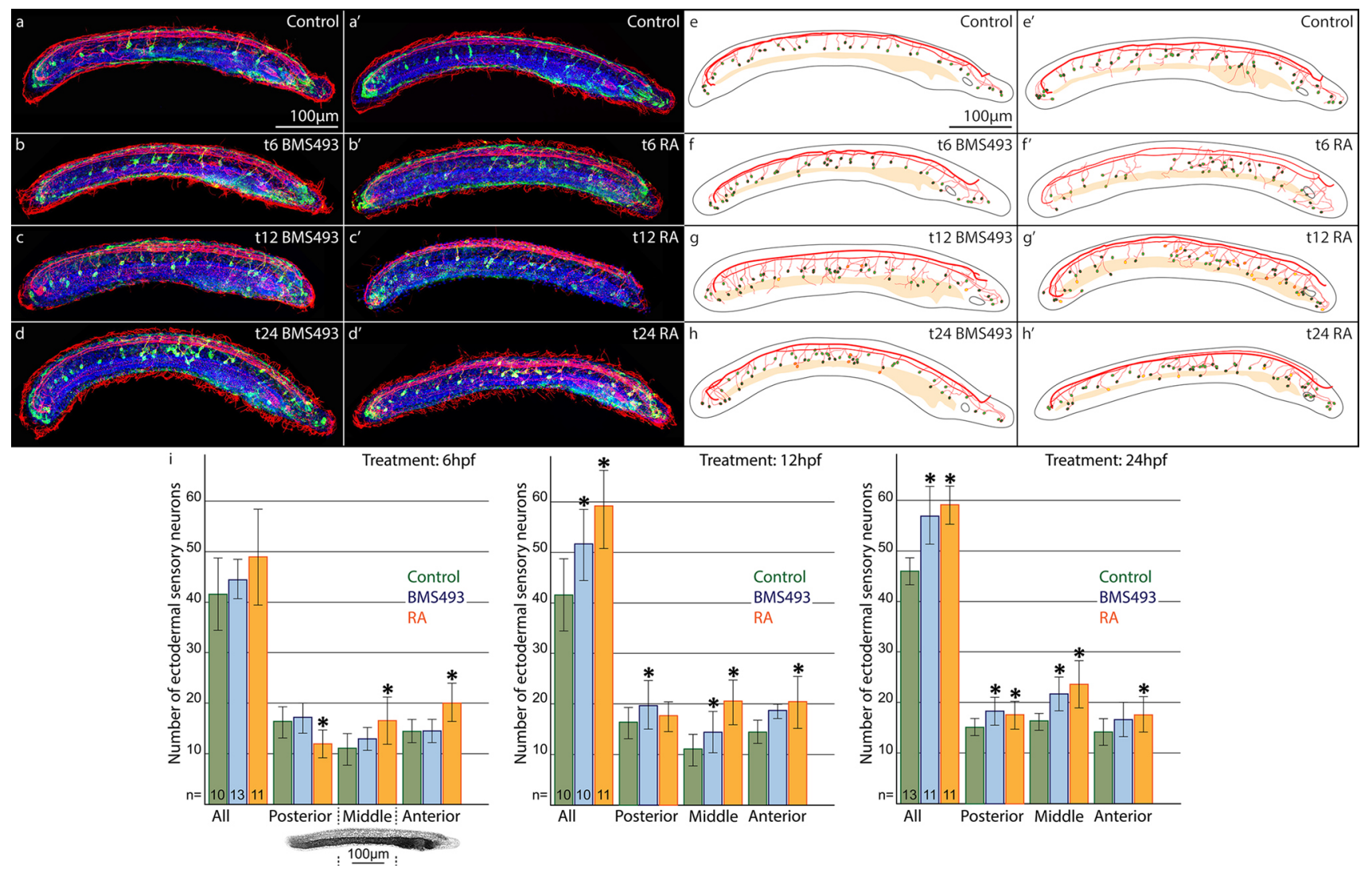




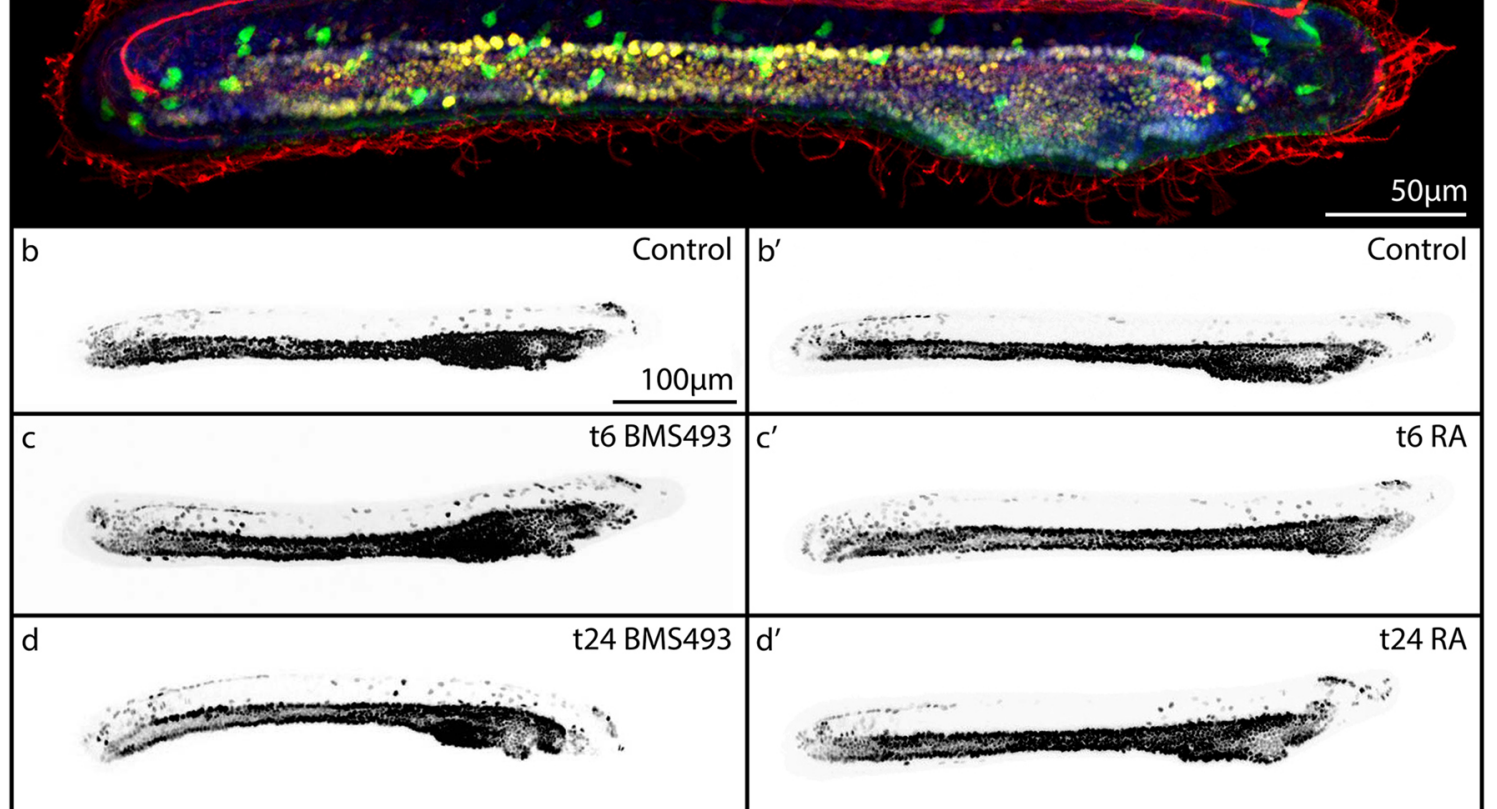

e

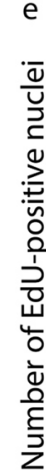

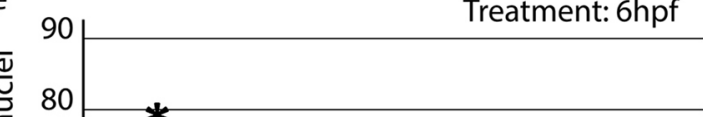

60

50

0 *

70 \begin{tabular}{|l}
\hline \\
\hline
\end{tabular}

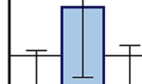

40
30

20

10

$\mathrm{n}=\mid$\begin{tabular}{l|l|l|l}
15 & 13 & 15
\end{tabular}

All
Treatment: $6 \mathrm{hpf}$

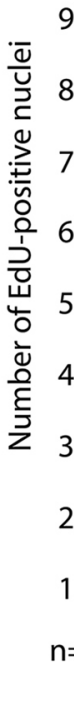

Treatment: 24hpf

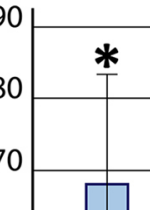

Control BMS493

RA

*

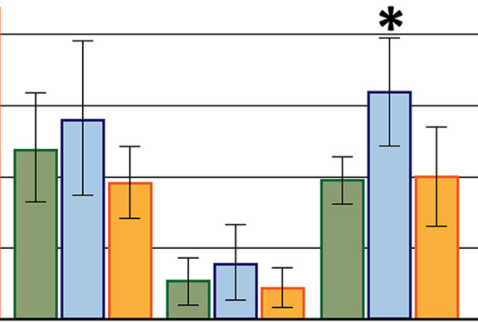

Posterior
Anterior 


\section{$100 \mu \mathrm{m}$}
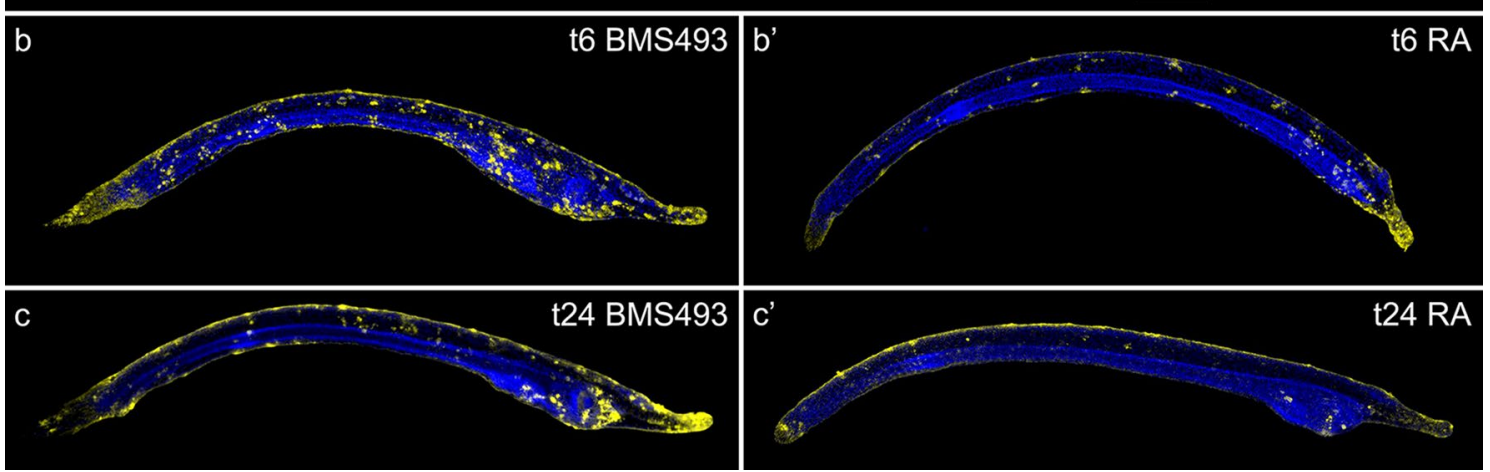


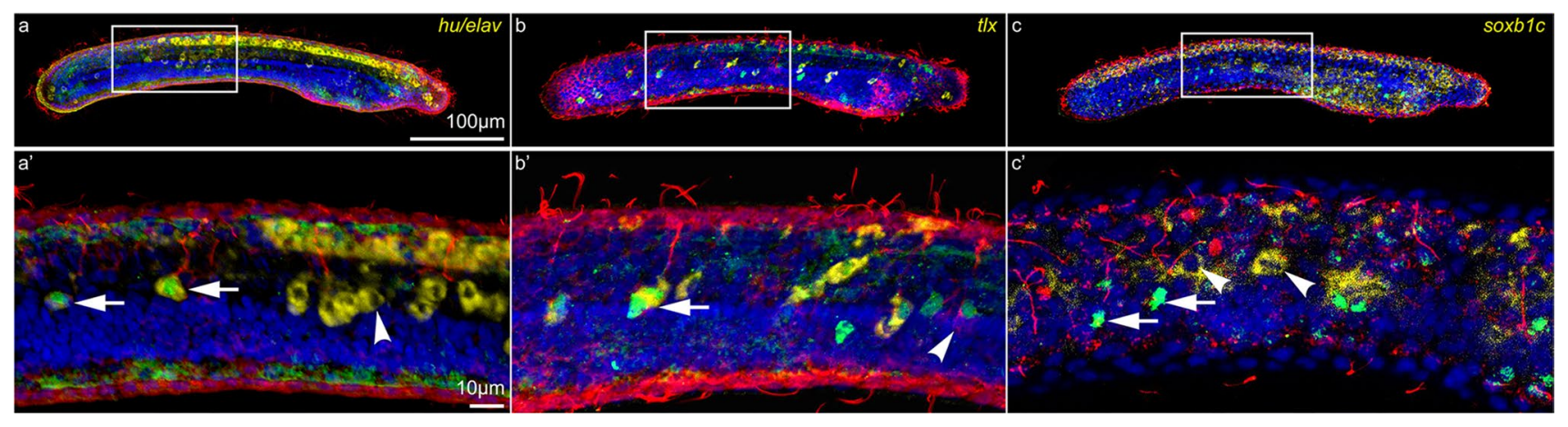



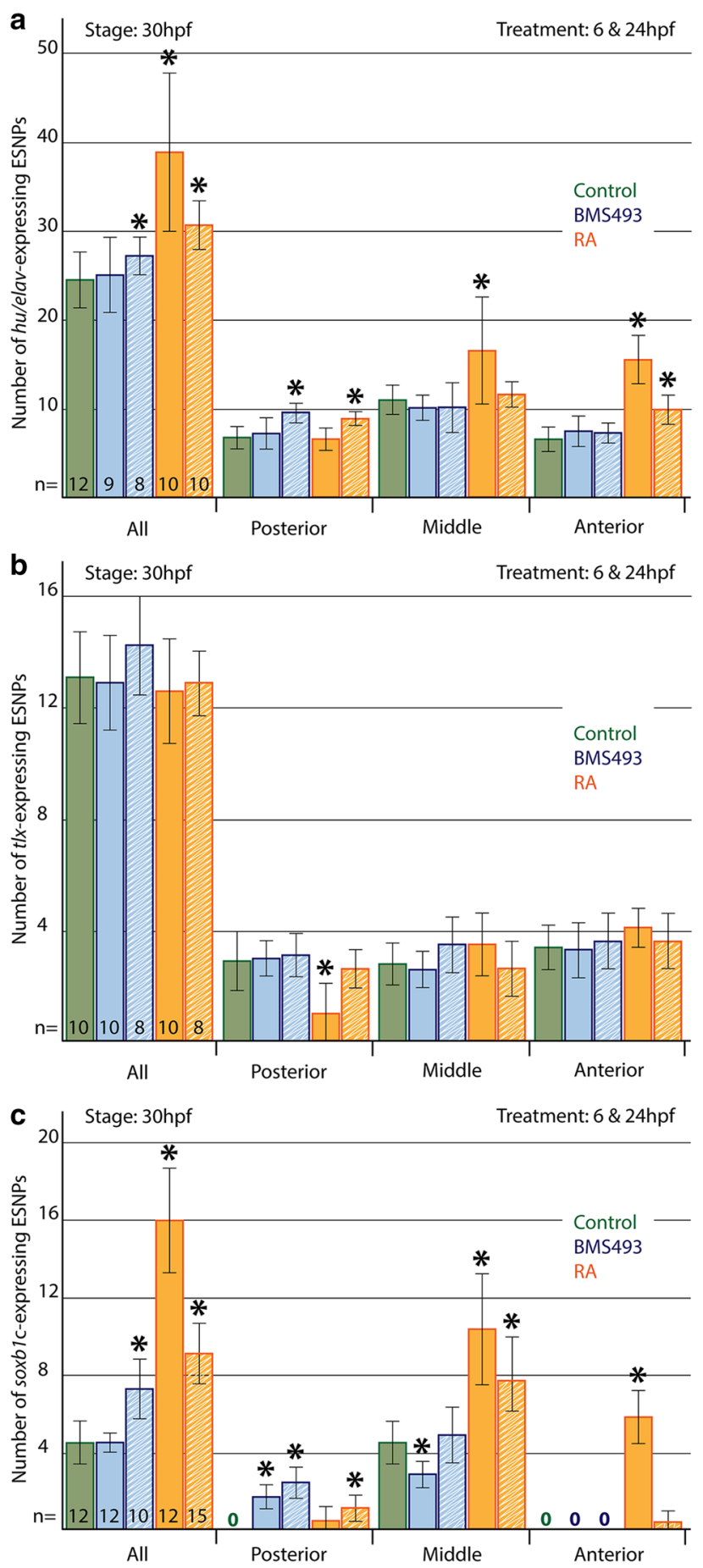

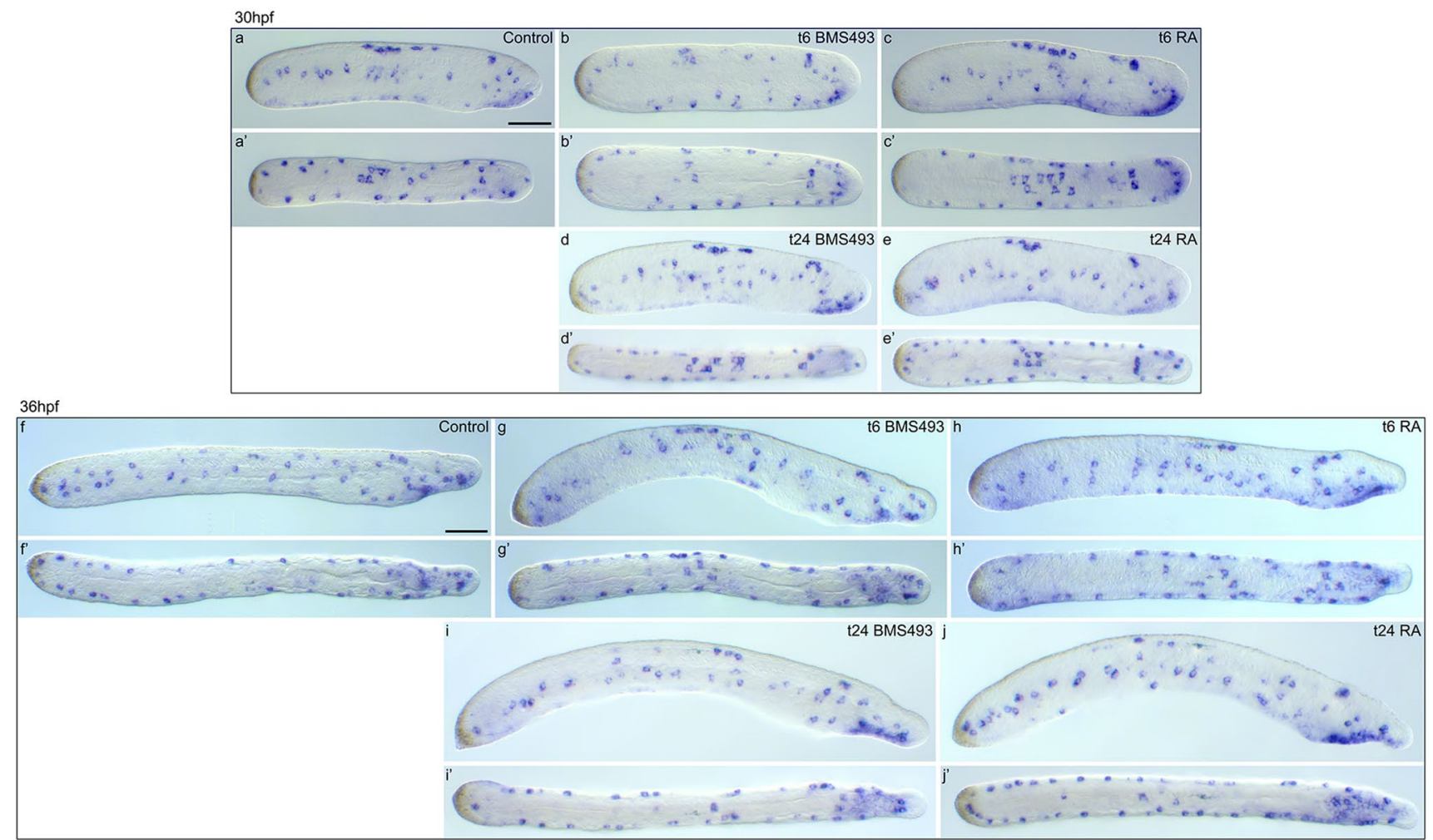
RA

\section{rar}

Ru/อU@v tIx soxb1c

000

0

$0 \quad 00$

0000

0

$0_{0}^{0} \begin{array}{llll}0 & 0 & 0 & 0\end{array}$

00 